\title{
Determining the Higgs boson self-coupling at hadron colliders
}

\author{
U. Baur* \\ Department of Physics, State University of New York, Buffalo, New York 14260 \\ T. Plehn ${ }^{\dagger}$ \\ CERN Theory Group, CH-1211 Geneva 23, Switzerland \\ D. Rainwater \\ DESY Theorie, Notkestrasse 85, D-22603 Hamburg, Germany \\ and Theory Group, Fermi National Accelerator Laboratory, Batavia, Illinois 60510 \\ (Received 14 November 2002; published 26 February 2003)
}

\begin{abstract}
Inclusive standard model Higgs boson pair production at hadron colliders has the capability to determine the Higgs boson self-coupling $\lambda$. We present a detailed analysis of the $g g \rightarrow H H \rightarrow\left(W^{+} W^{-}\right)\left(W^{+} W^{-}\right)$ $\rightarrow\left(j j \ell^{ \pm} \nu\right)\left(j j \ell^{\prime \pm} \nu\right)$ and $g g \rightarrow H H \rightarrow\left(W^{+} W^{-}\right)\left(W^{+} W^{-}\right) \rightarrow\left(j j \ell^{ \pm} \nu\right)\left(\ell^{\prime \pm} \nu \ell^{\prime \prime} \nu\right) \quad\left(\ell, \ell^{\prime}, \ell^{\prime \prime}=e, \mu\right) \quad$ signal channels, and the relevant background processes, for the CERN Large Hadron Collider, and a future Very Large Hadron Collider operating at a center-of-mass energy of $200 \mathrm{TeV}$. We also derive quantitative sensitivity limits for $\lambda$. We find that it should be possible at the LHC with design luminosity to establish that the standard model Higgs boson has a nonzero self-coupling and that $\lambda / \lambda_{S M}$ can be restricted to a range of $0-3.8$ at $95 \%$ confidence level (C.L.) if its mass is between 150 and $200 \mathrm{GeV}$. At a $200 \mathrm{TeV}$ collider with an integrated luminosity of $300 \mathrm{fb}^{-1}, \lambda$ can be determined with an accuracy of $8-25 \%$ at $95 \%$ C.L. in the same mass range.
\end{abstract}

DOI: 10.1103/PhysRevD.67.033003

PACS number(s): 14.80.Bn, 13.85.Qk

\section{INTRODUCTION}

The CERN Large Hadron Collider (LHC) is widely regarded as capable of directly observing the agent responsible for electroweak symmetry breaking and fermion mass generation. This is generally believed to be a light Higgs boson with mass $m_{H}<200 \mathrm{GeV}$ [1], a mass region for which discovery is covered by multiple channels, most notably by the decay that will be of interest here, $H \rightarrow W^{+} W^{-}$[2]. Once a Higgs boson candidate has been observed, emphasis will shift to a precise determination of its properties. The LHC promises complete coverage of Higgs boson decay scenarios [3], including general parametrizations in the minimal supersymmetric standard model (MSSM) $[3,4]$, invisible Higgs decays [5], and possibly even Higgs boson decays to muons [6]. With mild theoretical assumptions and an integrated luminosity of $200 \mathrm{fb}^{-1}$, the Higgs boson total width, $\Gamma_{H}$, and the gauge and various Yukawa couplings can be determined [7-9] with a precision of $10-30 \%$ [10]. At an $e^{+} e^{-}$linear collider with a center of mass energy of $350 \mathrm{GeV}$ or more, these measurements can be improved by up to a factor 10 [11], if an integrated luminosity of $500 \mathrm{fb}^{-1}$ can be achieved.

While these studies have shown that future colliders promise broad and significant capability to measure various properties of the Higgs sector, what remains is to determine the actual Higgs potential. Phenomenologically one should write an effective Lagrangian that does not already assume SM couplings, as the object at hand could be a radion or

\footnotetext{
*Email address: baur@ubhex.physics.buffalo.edu

†Email address: tilman.plehn@cern.ch

\#Email address: rain@mail.desy.de
}

other Higgs boson-like field that has different tree-level selfcouplings. Only after the potential is measured can it be decided what the candidate actually is. We take the Lagrangian as the effective potential

$$
V\left(\eta_{H}\right)=\frac{1}{2} m_{H}^{2} \eta_{H}^{2}+\lambda v \eta_{H}^{3}+\frac{1}{4} \tilde{\lambda} \eta_{H}^{4}
$$

where $\eta_{H}$ is the physical Higgs field, $v=\left(\sqrt{2} G_{F}\right)^{-1 / 2}$ is the vacuum expectation value, and $G_{F}$ is the Fermi constant. In the standard model (SM),

$$
\tilde{\lambda}=\lambda=\lambda_{S M}=\frac{m_{H}^{2}}{2 v^{2}} .
$$

Anomalous Higgs boson self-couplings appear in various beyond the SM scenarios, such as models with a composite Higgs boson [12], or in two Higgs doublet models, for example the MSSM [13], in which case one would use a different effective potential to measure deviations from the SM values for $\lambda$ and $\tilde{\lambda}$. Regarding the SM as an effective theory, the Higgs boson self-couplings $\lambda$ and $\tilde{\lambda}$ are per se free parameters. $S$-matrix unitarity constrains $\tilde{\lambda}$ to $\tilde{\lambda} \leqslant 8 \pi / 3$ [14].

Strictly speaking, both the trilinear Higgs boson coupling $\lambda$ and the quartic coupling $\tilde{\lambda}$ have to be measured separately in order to fully determine the Higgs potential. While $\lambda$ can be measured in Higgs pair production, triple Higgs production is needed to probe $\tilde{\lambda}$. Since the cross sections for $H H H$ production processes are more than a factor $10^{3}$ smaller than those for Higgs pair production at $e^{+} e^{-}$linear colliders [15], and about an order of magnitude smaller at hadron colliders [16], the quartic Higgs boson coupling will likely remain 
elusive even at the highest collider energies and luminosities considered so far. In the following we therefore restrict ourselves to $\lambda$.

Several studies of Higgs pair production in $e^{+} e^{-}$collisions have been conducted over the past few years $[15,17-$ 19], and quantitative sensitivity limits for $\lambda$ have been derived for several proposed linear colliders with center of mass energies spanning the range from $\sqrt{s}=500 \mathrm{GeV}$ to 3 $\mathrm{TeV}$. For example, a study employing neural net techniques found that $\lambda$ can be measured with a precision of about $20 \%$ at a linear collider with $\sqrt{s}=500 \mathrm{GeV}$ and an integrated luminosity of $1 \mathrm{ab}^{-1}$, if $m_{H}=120 \mathrm{GeV}$ [18]. In contrast, the potential of the LHC to probe the Higgs boson self-coupling has begun to be explored only recently. A survey of Higgs pair production and background processes at an upgraded LHC, which would gather 20 times the amount of data expected in the first run (dubbed SLHC), was presented in Ref. [20]. In Ref. [21], we discussed the prospects for determining $\lambda$ at the LHC with design luminosity in Higgs pair production via gluon fusion and subsequent decay to same-sign dileptons via weak gauge $W^{ \pm}$bosons,

$$
g g \rightarrow H H \rightarrow\left(W^{+} W^{-}\right)\left(W^{+} W^{-}\right) \rightarrow\left(j j \ell^{ \pm} \nu\right)\left(j j \ell^{\prime \pm} \nu\right),
$$

where $\ell$ and $\ell^{\prime}$ are any combination of electrons and muons, and presented quantitative estimates of sensitivity limits for $\lambda$ for $150 \mathrm{GeV} \leqslant m_{H} \leqslant 200 \mathrm{GeV}$ and various integrated luminosities. Finally, in Ref. [22], a PYTHIA [23] based study of several final states resulting from $g g \rightarrow H H$ $\rightarrow\left(W^{+} W^{-}\right)\left(W^{+} W^{-}\right) \quad$ and $\quad g g \rightarrow H H \rightarrow\left(W^{+} W^{-}\right)(Z Z)$ for LHC and SLHC was conducted.

In this paper we present a more detailed and extended analysis of Higgs pair production via gluon fusion at hadron colliders. In Ref. [21], we included only the two largest sources of background, $W^{ \pm} W^{+} W^{-} j j$ and $t \bar{t} W^{ \pm}$production, in our calculation. The effect of the remaining background contributions on the sensitivity limits for $\lambda$ was estimated by scaling the combined $W W W j j$ and $t \bar{t} W$ cross section by a factor 1.1, as suggested by Ref. [20], which found that the remaining backgrounds are small. Here we present a more complete calculation of the background which includes, in addition to $W W W j j$ and $t \bar{t} W$ production, $W^{ \pm} W^{ \pm} j j j j$, $W^{ \pm} Z j j j j, t \bar{t} Z, t \bar{t} j, t \bar{t} t \bar{t}, W^{+} W^{-} W^{+} W^{-}$and $W W Z j j$ production. Furthermore, we discuss the potential size of backgrounds arising from overlapping events and double-parton scattering. We also extend our previous analysis by considering the three lepton channel

$$
g g \rightarrow H H \rightarrow\left(W^{+} W^{-}\right)\left(W^{+} W^{-}\right) \rightarrow\left(j j \ell^{ \pm} \nu\right)\left(\ell^{\prime \pm} \nu \ell^{\prime \prime \mp} \nu\right)
$$

$\left(\ell, \ell^{\prime}, \ell^{\prime \prime}=e, \mu\right)$, and the background processes which affect it. In addition to the LHC and SLHC, we calculate signal and background cross sections, and derive sensitivity bounds for $\lambda$, for a Very Large Hadron Collider (VLHC), assuming a $p p$ collider operating at $\sqrt{s}=200 \mathrm{TeV}$ with a luminosity of $\mathcal{L}$ $=2 \times 10^{34} \mathrm{~cm}^{-2} \mathrm{~s}^{-1}$. These parameters correspond to one of the options listed in Ref. [24].
The remainder of this paper is organized as follows. In Secs. II and III we outline our calculation of signal and background processes for the same-sign dilepton and three lepton final states, respectively. In Sec. IV, we derive sensitivity limits for $\lambda$ for various integrated luminosities at the LHC and VLHC. Our conclusions are given in Sec. V.

\section{THE SAME-SIGN DILEPTON FINAL STATE}

There are several mechanisms for pair production of Higgs bosons in hadronic collisions. Higgs boson pairs can be produced via gluon fusion, $g g \rightarrow H H[16,25]$, weak boson fusion, $q q \rightarrow q q H H$ [26], associated production with $W$ or $Z$ bosons, $q \bar{q} \rightarrow V H H, V=W, Z$ [27], and associated production with $t \bar{t}$ pairs, $g g, q \bar{q} \rightarrow t \bar{t} H H$ [20]. At the LHC, inclusive Higgs boson pair production is dominated by gluon fusion. The weak boson fusion process, and associated production with $W / Z$ bosons or $t \bar{t}$ pairs yield cross sections which are about a factor 10 and 30 smaller than that for $g g$ $\rightarrow H H[25,20]$. Since Higgs pair production at the LHC is rate limited, we concentrate on the gluon fusion process in the following.

For $m_{H}<140 \mathrm{GeV}$, the dominant decay mode of the SM Higgs boson is $H \rightarrow b \bar{b}$, and the QCD $b \bar{b} b \bar{b}$ background overwhelms the $g g \rightarrow H H$ signal [28]. For $m_{H}>140 \mathrm{GeV}$, $H \rightarrow W^{+} W^{-}$dominates, and the $W^{+} W^{-} W^{+} W^{-}$final state has the largest individual branching ratio. If all $W$ bosons decay hadronically, QCD multi-jet production dwarfs the signal. A similar result is obtained for the $\ell^{ \pm} \nu+6$ jet (only one $W$ boson decays leptonically), and $\ell^{ \pm} \nu \ell^{\prime \mp} \nu+4$ jet (one $W^{+} W^{-}$pair decays leptonically) final states, where $W+$ multi-jet and $W^{+} W^{-}+$multi-jet production provide very large backgrounds. This leaves the same-sign dilepton final states, $\left(j j \ell^{ \pm} \nu\right)\left(j j \ell^{\prime \pm} \nu\right)$, modes where three $W$ bosons decay leptonically and one decays hadronically, and the allleptonic decay modes. The latter suffer from a large suppression due to the small $W W W W \rightarrow 4 \ell+4 \nu$ branching ratio of $(0.216)^{4}=0.0022(\mathrm{BR}(W \rightarrow \ell \nu)=0.216, \ell=e, \mu)$. In the following we therefore only consider the $\left(j j \ell^{ \pm} \nu\right)\left(j j \ell^{\prime \pm} \nu\right)$ and $\left(j j \ell^{ \pm} \nu\right)\left(\ell^{\prime \pm} \nu \ell^{\prime \prime \mp} \nu\right)$ final states.

In this section we discuss in detail the calculation of signal and background cross sections for the $\left(j j \ell^{ \pm} \nu\right)\left(j j \ell^{\prime \pm} \nu\right)$ final state. The three lepton final state will be considered in Sec. III.

\section{A. Calculation of the signal cross section}

The Feynman diagrams contributing to $g g \rightarrow H H$ in the SM consist of fermion triangle and box diagrams (see Fig. 1) [16]. Non-standard Higgs boson self-couplings only affect the triangle diagrams with a Higgs boson exchanged in the $s$-channel. We calculate the $g g \rightarrow H H \rightarrow\left(W^{+} W^{-}\right)\left(W^{+} W^{-}\right)$ $\rightarrow\left(j j \ell^{ \pm} \nu\right)\left(j j \ell^{\prime \pm} \nu\right)$ cross section using exact loop matrix elements [16]. As demonstrated in Ref. [21], the infinite top quark mass limit, which is commonly used in place of exact matrix elements to speed up the calculation, reproduces the correct total cross section for $\mathrm{HH}$ production to within $10 \%$ to $30 \%$ for Higgs boson masses between $140 \mathrm{GeV}$ and 200 

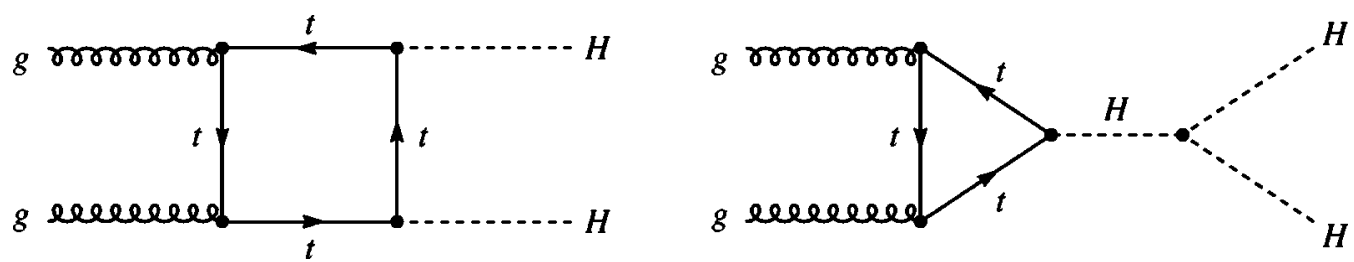

FIG. 1. Representative Feynman diagrams for the process $g g \rightarrow H H$.

$\mathrm{GeV}$, but produces completely incorrect kinematic distributions. The intermediate Higgs and $W$ bosons are treated offshell using finite widths in the double pole approximation in our calculation. Decay correlations for the $H \rightarrow W^{+} W^{-} \rightarrow 4$ fermion decays are fully taken into account [29].

Signal results are computed consistently to leading order QCD with the top quark mass set to $m_{t}=175 \mathrm{GeV}$ and SM $H W W$ and top quark Yukawa couplings, and the renormalization and factorization scales are taken to be the Higgs boson mass [16]. The contributions of all other quarks to the $g g \rightarrow H H$ box and triangular diagrams are strongly suppressed due to their $H f \bar{f}$ couplings, which are proportional to the fermion mass. The effects of next-to-leading (NLO) order QCD corrections are included in our calculation by multiplying the differential cross section by an overall factor $K$ $=1.65$ at LHC and $K=1.35$ at VLHC energies ( $K$ factor) for scale choice $\mu=m_{H}$, as suggested by Ref. [30] where the QCD corrections for $g g \rightarrow H H$ have been computed in the large $m_{t}$ limit. Although this approximation cannot replace a calculation of the full NLO QCD corrections to $g g \rightarrow H H$, it is expected to work well in this particular case: it is well known from single Higgs boson production via gluon fusion [31] that the two-loop QCD corrections to the one-loop Higgs production amplitude are well approximated by multiplying the leading order one-loop cross section for a finite top quark mass by the $K$ factor obtained in the large $m_{t}$ limit. This feature can be easily understood by recalling that the dominant corrections originate from radiation off the initial state gluons, which is universal.

The lowest order $g g \rightarrow H H$ cross section exhibits a rather strong dependence on the renormalization and factorization scales. For example, for $m_{H}=200 \mathrm{GeV}$ and $\mu^{2}=m_{H}^{2}$, and using exact loop matrix elements, one obtains a total cross section of $8.26 \mathrm{fb}$. For $\mu^{2}=\hat{s}$, on the other hand, one finds a cross section which is almost a factor 1.5 smaller. NLO QCD corrections, albeit computed in the large $m_{t}$ limit, significantly reduce the $\mu$ dependence: varying the scale $\mu$ from $\mu^{2}=m_{H}^{2}$ to $\mu^{2}=\hat{s}$, the cross section including the $K$ factor decreases by a factor 1.25 instead of 1.5 without the $K$ factor.

For $\mu^{2}=m_{H}^{2}$, the $K$ factor at VLHC energies is smaller than that obtained for the LHC. At higher energies, smaller parton momentum fractions are probed. This results in an increased sensitivity of the cross section to the choice of factorization scale which partially compensates the variation of the cross section with the renormalization scale.

In all our calculations we use a value for the strong coupling constant of $\alpha_{s}\left(M_{Z}\right)=0.1185$ [32]. All signal and background cross sections are computed using CTEQ4L [33] parton distribution functions.
The kinematic acceptance cuts for both signal and backgrounds in the $\left(j j \ell^{ \pm} \nu\right)\left(j j \ell^{\prime \pm} \nu\right)$ channel at the LHC and VLHC are

$$
\begin{gathered}
p_{T}(j)>30,30,20,20 \mathrm{GeV}, \quad p_{T}(\ell)>15,15 \mathrm{GeV}, \\
|\eta(j)|<3.0, \quad|\eta(\ell)|<2.5, \\
\Delta R(j j)>0.6, \quad \Delta R(j \ell)>0.4, \quad \Delta R(\ell \ell)>0.2,
\end{gathered}
$$

where $\Delta R=\left[(\Delta \phi)^{2}+(\Delta \eta)^{2}\right]^{1 / 2}$ is the separation in the pseudorapidity-azimuthal angle plane. In addition we require the four jets to combine into two pseudo- $W$ pairs with invariant masses

$$
50 \mathrm{GeV}<m(j j)<110 \mathrm{GeV},
$$

and assume that this captures $100 \%$ of the signal and backgrounds. We do not impose a missing transverse momentum cut which would remove a considerable fraction of the signal events. Detector resolution effects are not taken into account in our calculation.

As we have shown in Ref. [21], at the LHC, the main background from $W W W j j$ and $t \bar{t} W$ production can be reduced by about $45 \%$, with little impact on the signal, by imposing a more restrictive jet-jet separation cut of $\Delta R(j j)$ $>1.0$. In contrast, at VLHC energies, there is little gain in tightening the $\Delta R(j j)$ cut. Subsequently, we therefore require

$$
\Delta R(j j)>1.0 \quad \text { at the LHC, }
$$

and

$$
\Delta R(j j)>0.6 \quad \text { at the VLHC }
$$

in the $\left(j j \ell^{ \pm} \nu\right)\left(j j \ell^{\prime \pm} \nu\right)$ channel.

Our choice of $p_{T}$ cuts for jets and leptons in Eqs. (5)-(7) is driven by the goal of retaining as much signal as possible while ensuring that the LHC experiments, ATLAS and CMS, can record $\ell^{ \pm} \ell^{\prime \pm}+4 j$ events when operating at the LHC design luminosity of $\mathcal{L}=10^{34} \mathrm{~cm}^{-2} \mathrm{~s}^{-1}$. Figures 2 and 3 show the $p p \rightarrow H H \rightarrow\left(W^{+} W^{-}\right)\left(W^{+} W^{-}\right) \rightarrow \ell^{ \pm} \ell^{\prime \pm}+4 j$ differential cross section at the LHC as a function of the lepton and jet minimum transverse momentum, respectively. Qualitatively similar results are obtained at VLHC energies. The differential cross section peaks at low values of $p_{T \text { min }}$ and falls very quickly with increasing values of the minimum transverse momentum, in particular in the jet case. In order to maximize the signal cross section, the lepton and jet $p_{T}$ thresholds are thus chosen as low as possible and yet be 


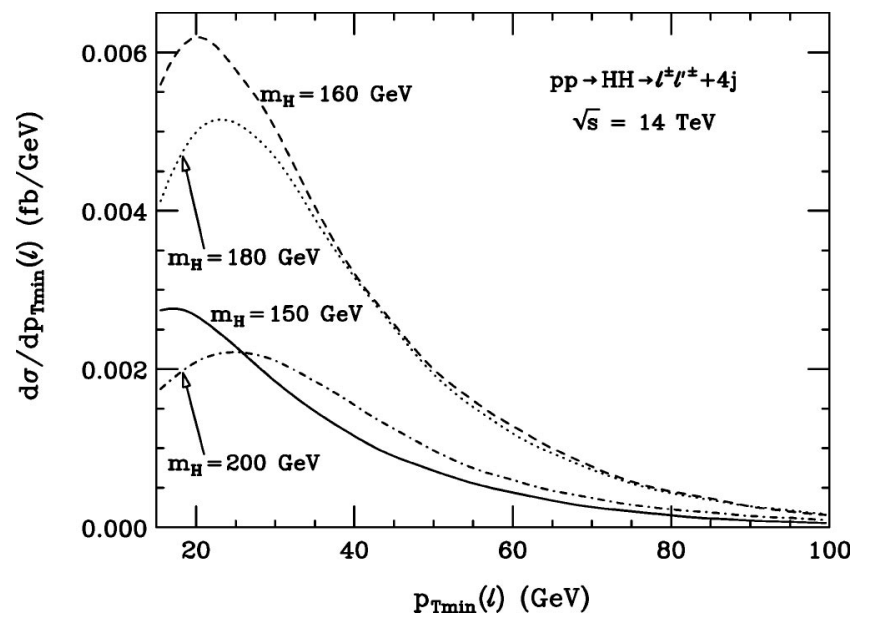

FIG. 2. The $p p \rightarrow H H \rightarrow\left(W^{+} W^{-}\right)\left(W^{+} W^{-}\right) \rightarrow \ell^{ \pm} \ell^{\prime \pm}+4 j$ differential cross section as a function of the minimum lepton transverse momentum, $p_{\text {Tmin }}(\ell)$, for $p p$ collisions at $\sqrt{s}=14 \mathrm{TeV}$. Results are shown for $m_{H}=150 \mathrm{GeV}$ (solid line), $m_{H}=160 \mathrm{GeV}$ (dashed line), $m_{H}=180 \mathrm{GeV}$ (dotted line), and $m_{H}=200 \mathrm{GeV}$ (dash-dotted line).

compatible with the requirements of ATLAS and CMS to successfully record such events.

We shall also use the cuts listed in Eqs. (5)-(7) for a luminosity upgraded LHC operating at $\mathcal{L}=10^{35} \mathrm{~cm}^{-2} \mathrm{~s}^{-1}$, and at the VLHC. Preliminary studies have concluded $[20,34]$ that cuts similar to those listed in Eq. (5) - (7) should be sufficient, although increased background from event pileup is expected to degrade detector performance, in particular at the SLHC.

\section{B. Calculation of the backgrounds}

The SM backgrounds of interest are those that produce two same-sign leptons and four well-separated jets which



FIG. 3. The $p p \rightarrow H H \rightarrow\left(W^{+} W^{-}\right)\left(W^{+} W^{-}\right) \rightarrow \ell^{ \pm} \ell^{\prime \pm}+4 j$ differential cross section as a function of the minimum jet transverse momentum, $p_{\text {Tmin }}(j)$, for $p p$ collisions at $\sqrt{s}=14 \mathrm{TeV}$. Results are shown for $m_{H}=150 \mathrm{GeV}$ (solid line), $m_{H}=160 \mathrm{GeV}$ (dashed line), $m_{H}=180 \mathrm{GeV}$ (dotted line), and $m_{H}=200 \mathrm{GeV}$ (dash-dotted line). reconstruct in two pairs to a window around the $W$ boson mass. The largest contribution originates from $W^{ \pm} W^{+} W^{-} j j$ production, followed by $t \bar{t} W^{ \pm}$where one top quark decays leptonically, the other hadronically, and neither $b$ quark jet is tagged. Other backgrounds which contribute are: $W^{ \pm} W^{ \pm} j j j j$ production; $t \bar{t} t \bar{t}$ production, where none of the $b$ quark jets are tagged, and additional jets or leptons are not observed; $W^{ \pm} Z j j j j, t \bar{t} Z$ and $W^{+} W^{-} Z j j$ production with leptonic $Z$ decay (including off-shell photon interference) where one lepton is not observed; and $t \bar{t} j$ events where one $b$ quark decays semileptonically with good hadronic isolation, and the other is not tagged. In addition, in a high luminosity environment, one has to worry about backgrounds from overlapping events and double parton scattering. $b$ quarks are assumed to be tagged with an efficiency of 50\% throughout. We do not apply an explicit $K$ factor for the backgrounds here; however, we do later include the potential effect of QCD corrections on the backgrounds when we extract limits on the Higgs self-coupling (see Sec. IV).

\section{The $W W W j j, t \bar{t} W, W^{+} W^{-} W^{+} W^{-}$and $t \bar{t} t \bar{t}$ backgrounds}

We simulate these backgrounds at the parton level using exact matrix elements generated with MADGRAPH [35]. The $W W W j j$ background has a significant contribution from $W H\left(\rightarrow W^{+} W^{-}\right) j j$ production. For $W W W j j$ production we evaluate the strong coupling constant $\alpha_{s}$ and the parton distribution functions at a scale $\mu$ given by $\mu^{2}=\Sigma p_{T}^{2}$, where the sum extends over all final state particles; for $t \bar{t} W$ production we take $\mu=m_{t}+M_{W} / 2$, and in the $t \bar{t} t \bar{t}$ case we use $\mu=2 m_{t}$. Top quarks are generated on shell (narrow width approximation), while all $W$ bosons are allowed to be off shell. Events with one or more tagged $b$ quarks are rejected. In the $t \bar{t} t \bar{t}$ case we merge jets if their separation in the pseudorapidity-azimuthal angle plane is $\Delta R(j j)<0.6$. The $W^{+} W^{-} W^{+} W^{-}$background has a significant contribution from $W W H(\rightarrow W W)$ production. The $W^{+} W^{-} W^{+} W^{-}$cross section at the LHC (VLHC) is found to be a factor 5 to 25 (25 to 80) smaller than that of $W W W j j$ production, depending on the mass of the Higgs boson. In this analysis we therefore ignore the $W^{+} W^{-} W^{+} W^{-}$background.

\section{The $W^{ \pm} W^{ \pm} j j j j$ background}

Although MADGRAPH is able to generate exact matrix elements for $W^{ \pm} W^{ \pm} j j j j$ production, the large number of contributing Feynman diagrams (more than 6000) makes a full matrix element based calculation of the $W^{ \pm} W^{ \pm} j j j j$ background impractical. In order to estimate the $W^{ \pm} W^{ \pm} j j j j$ cross section we thus have interfaced the matrix elements for $p p \rightarrow W^{ \pm} W^{ \pm} j j[36]$ with PYTHIA, which produces the two additional jets in a leading-log shower approximation. The strong coupling constant $\alpha_{s}$ and the parton distribution functions are evaluated at a scale $\mu$ given by $\mu^{2}=\Sigma p_{T}^{2}$, where the sum extends over all final state particles.

\section{The tit $Z, W^{ \pm} Z j j j j$ and $W W Z j j$ backgrounds}

We calculate the $t \bar{t} Z$ cross section using exact matrix elements. Since more than 15000 Feynman diagrams contrib- 
ute to $W^{ \pm} Z j j j j$ production, we estimate the cross section for this process by interfacing the $W^{ \pm} Z j j$ matrix elements [37] with PYTHIA, similar to $W^{ \pm} W^{ \pm} j j j j$ production. Off-shell photon interference effects are taken into account in both cases. Both processes contribute to the background only if one of the leptons from $Z$ or off-shell photon decay is missed. We consider a lepton to be missed if it has $p_{T}$ $<10 \mathrm{GeV}$ or $|\eta|>2.5$. If the lepton is within a cone of $\Delta R<0.2$ from a detected lepton and has $1 \mathrm{GeV}<p_{T}$ $<10 \mathrm{GeV}$, then the detected lepton is not considered isolated and the event is rejected. The strong coupling constant and the parton distribution functions are evaluated in $t \bar{t} Z$ $\left(W^{ \pm} Z j j j j\right)$ production at $\mu=m_{t}+M_{Z} / 2\left(\mu^{2}=\Sigma p_{T}^{2}\right)$. In order to avoid the collinear singularity when the missed lepton is collinear with an observed lepton (which is only relevant if the missed lepton has $p_{T}<1 \mathrm{GeV}$ ), finite lepton masses must be maintained in the calculation of the $W^{ \pm} Z j j j j$ and $t \bar{t} Z$ processes [9], the latter also including additional considerations to maintain gauge invariance of the calculation, due to finite top quark width which must be included for these events. $t \bar{t} Z$ events are rejected with a factor 4 , which approximates the fraction of events with one or more tagged $b$ quarks.

The size of the $W W Z j j$ background can be estimated from the ratio of the $W W Z$ and $W W W$ cross sections, together with the suppression factor which arises from requiring that one lepton is missed, and the $W W W j j$ rate. We find that the $W W Z j j$ cross section is about a factor 30 to 60 smaller than the $W W W j j$ cross section, depending on the mass of the Higgs boson. We thus ignore the $W W Z j j$ background in the following.

\section{The $t \bar{t} j$ background}

We calculate $t \bar{t} j \rightarrow W^{+} b W^{-} \bar{b} j$ production where one of the $b$-quarks decays semileptonically with an isolated lepton using exact $t \bar{t}+$ parton and $b \rightarrow c \ell \nu$ matrix elements. The lepton from $b$ decay is considered not isolated if the charm quark is within a cone of $\Delta R<0.4$ from the lepton and has $p_{T}(c)>3 \mathrm{GeV}$. Events are rejected with a factor two, which approximates the fraction where the second $b$ quark would be tagged. In order to regularize the soft parton $p_{T}$ distribution so as to reproduce the $p_{T}$ distribution of the hard recoil system $(t \bar{t})$ from a full resummation calculation [38], while preserving the normalization of the hard $t \bar{t}$ cross section, we use the truncated shower approximation (TSA) [39]. The advantage is that QCD matrix elements at tree level contain the full information on angular distributions and hardness of additional jet emission. A parton shower approach as used in Ref. [22] would not immediately give reliable answers unless both color coherence and the choice of scale are implemented correctly, matching the answer given by QCD matrix elements for sufficiently hard partons. In practice this is achieved by integrating the full tree-level $t \bar{t}+$ parton matrix elements over phase space down to $p_{T}$ (parton) $>1 \mathrm{GeV}$, and multiplying the result by a factor $1-\exp$ $\left[-p_{T}^{2}\right.$ (parton) $\left./ p_{T S A}^{2}\right]$, where $p_{T S A}$ is adjusted to achieve $\sigma_{t \bar{t} j}$ $=\sigma_{t \bar{t}}$. For a scale choice of $\mu^{2}=m_{t}^{2}$, we find $p_{T S A}$
TABLE I. Luminosity, bunch spacing, and luminosity per bunch crossing for the LHC [20], SLHC [20] and VLHC [24].

\begin{tabular}{lccc}
\hline \hline Machine & Luminosity $\mathcal{L}$ & Bunch spacing $\Delta \tau$ & $\mathcal{L}_{b c}$ \\
\hline LHC & $10^{34} \mathrm{~cm}^{-2} \mathrm{~s}^{-1}$ & $25 \mathrm{~ns}$ & $(4.0 \mathrm{mb})^{-1}$ \\
SLHC & $10^{35} \mathrm{~cm}^{-2} \mathrm{~s}^{-1}$ & $12.5 \mathrm{~ns}$ & $(0.8 \mathrm{mb})^{-1}$ \\
VLHC & $2 \times 10^{34} \mathrm{~cm}^{-2} \mathrm{~s}^{-1}$ & $18.8 \mathrm{~ns}$ & $(2.7 \mathrm{mb})^{-1}$ \\
\hline \hline
\end{tabular}

$=15,50 \mathrm{GeV}$ for the LHC and VLHC, respectively. This should not be construed as an attempt to mimic a full NLO calculation of $t \bar{t} j$ production, which is not available, but it does represent an improvement over using only PYTHIA $t \bar{t}$ matrix elements with additional partonic emission from showering, and includes a well-motivated approach to controlling the soft singularity present in the exact $\mathcal{O}\left(\alpha_{s}^{3}\right)$ matrix elements.

\section{Other backgrounds: Overlapping events and double parton scattering}

At a high-luminosity intersection point of colliding beams, more than one event may occur per bunch crossing. In principle, such overlapping events can be recognized by a total visible energy measurement or by tracing some final particle tracks back to distinct event vertices, but in practice this may not always be possible. In this case, the overlap of, e.g, two single $H \rightarrow W^{+} W^{-}$events, a $W^{+} W^{-}$and a $W j j$ event, or a $t \bar{t}$ and a single $W$ event may constitute a potentially dangerous source of background for the Higgs boson pair production signal. If the vertices of the overlapping events cannot be resolved, the effective cross section for overlapping events is given by [36]

$$
\sigma_{o v}(1,2)=\frac{1}{2} \sigma(1) \sigma(2) \mathcal{L}_{b c},
$$

where $\sigma(1)$ and $\sigma(2)$ are the cross sections for the two overlapping processes, and $\mathcal{L}_{b c}$ is the luminosity per bunch crossing. It is given by

$$
\mathcal{L}_{b c}=\mathcal{L} \Delta \tau
$$

where $\mathcal{L}$ is the instantaneous luminosity and $\Delta \tau$ is the bunch spacing. The values for $\mathcal{L}, \Delta \tau$ and $\mathcal{L}_{b c}$ at the LHC, SLHC and VLHC are listed in Table I.

The $\ell^{ \pm} \ell^{\prime \pm} j j j j$ final state can also be produced via the independent scattering of two pairs of partons in the incident protons. The cross section for double parton scattering is given by Eq. (11) with the factor $\mathcal{L}_{b c} / 2$ replaced by $1 / \sigma_{\text {eff }}$. The parameter $\sigma_{\text {eff }} \approx 15 \mathrm{mb}$ [40], the effective cross section, contains all the information about the non-perturbative structure of the proton in this simplified approach. It is believed that $\sigma_{\text {eff }}$ is largely independent of the center of mass energy [41]. Comparison of $\sigma_{\text {eff }}$ and the values for $\mathcal{L}_{b c}$ listed in Table I shows that the double parton cross section is about a factor 2 to 10 smaller than that from overlapping events. 
TABLE II. Higgs pair signal and background cross sections (fb) for $p p \rightarrow \ell^{ \pm} \ell^{\prime \pm}+4 j\left(\ell, \ell^{\prime}=e, \mu\right)$ at (a) the LHC $(\sqrt{s}=14 \mathrm{TeV})$ and (b) at the VLHC $(\sqrt{s}=200 \mathrm{TeV})$, imposing the cuts listed in Eqs. (5) $-(7)$, and as a function of the Higgs boson mass $(\mathrm{GeV})$. The background labeled "pileup" represents a rough estimate of the combined $W W W j j, t \bar{t} W, t \bar{t} Z, W Z j j j j, W W j j j j$ and $t \bar{t} t \bar{t}$ cross section from overlapping events and double parton scattering. Cross sections at the SLHC are identical to those in the LHC case with the exception of the pileup cross section, which is about a factor 3.7 larger than at the LHC. The last column, labeled $\mathcal{B}_{\text {tot }}$, shows the total background cross section.

\begin{tabular}{ccccccccccc}
\hline \hline \multicolumn{10}{c}{ (a) LHC } \\
$m_{H}$ & $H H$ & $W W W j j$ & $t \bar{t} W$ & $t \bar{t} Z$ & $t \bar{t} j$ & $W Z j j j j$ & $W W j j j$ & $t \bar{t} t \bar{t}$ & pileup & $\mathcal{B}_{\text {tot }}$ \\
\hline 150 & 0.07 & 0.36 & 0.22 & 0.05 & 0.08 & 0.15 & 0.005 & 0.002 & $\sim 0.03$ & 0.90 \\
160 & 0.19 & 0.49 & 0.22 & 0.05 & 0.08 & 0.15 & 0.005 & 0.002 & $\sim 0.03$ & 1.03 \\
180 & 0.18 & 0.40 & 0.22 & 0.05 & 0.08 & 0.15 & 0.005 & 0.002 & $\sim 0.03$ & 0.94 \\
200 & 0.08 & 0.29 & 0.22 & 0.05 & 0.08 & 0.15 & 0.005 & 0.002 & $\sim 0.03$ & 0.83 \\
& & & & & $(\mathrm{~b})$ VLHC & & & & \\
$m_{H}$ & $H H$ & $W W W j j$ & $t \bar{t} W$ & $t \bar{t} Z$ & $t \bar{t} j$ & $W Z j j j j$ & $W W j j j$ & $t \bar{t} t \bar{t}$ & pileup & $\mathcal{B}_{\text {tot }}$ \\
\hline 140 & 2.2 & \multirow{2}{*}{14.9} & 5.8 & 7.4 & 7.7 & 8.1 & 0.13 & 6.13 & $\sim 20$ & 70.2 \\
150 & 6.5 & 17.0 & 5.8 & 7.4 & 7.7 & 8.1 & 0.13 & 6.13 & $\sim 20$ & 72.3 \\
160 & 15.8 & 20.4 & 5.8 & 7.4 & 7.7 & 8.1 & 0.13 & 6.13 & $\sim 20$ & 75.7 \\
180 & 16.0 & 17.9 & 5.8 & 7.4 & 7.7 & 8.1 & 0.13 & 6.13 & $\sim 20$ & 73.2 \\
200 & 8.7 & 14.3 & 5.8 & 7.4 & 7.7 & 8.1 & 0.13 & 6.13 & $\sim 20$ & 69.6 \\
\hline \hline
\end{tabular}

\section{Numerical results}

The total cross sections within cuts [see Eqs. (5) - (7)] for signal and background processes at the LHC and VLHC are listed in Table II. At the LHC, with $300 \mathrm{fb}^{-1}$, at most about 50 signal events are produced. Outside of the Higgs boson mass range considered here, the number of signal events is too small to be useful. For $m_{H}<150 \mathrm{GeV}$, this is due to the small $H \rightarrow W^{*} W$ branching ratio. For $m_{H}>200 \mathrm{GeV}$, the $g g \rightarrow H H$ cross section is too small. $W W W j j$ and $t \bar{t} W$ production are the largest contributions to the background. The background from $t \bar{t} Z$ production where one of the leptons is lost is moderate. Although the cross section for $W Z j j j j$ production is substantial, this background can be separated rather easily from the signal as discussed below. $W W j j j j$ and $t \bar{t} t \bar{t}$ production contribute negligibly to the background at the LHC. The $t \bar{t} t \bar{t}$ cross section is suppressed by the large top quark mass. The $W W j j j j$ cross section is small because quark-gluon and gluon-gluon fusion processes do not contribute to same sign $W$ pair production.

The $t \bar{t} j$ cross section is extremely sensitive to the lepton $p_{T}$ cut imposed. Requiring $p_{T}(\ell)>15 \mathrm{GeV}$, the $t \bar{t} j$ background is of the same size as the $t \bar{t} Z$ background, and about a factor 3 smaller than the $t \bar{t} W$ background. Decreasing the $p_{T}(\ell)$ cut to $10 \mathrm{GeV}$, the $t \bar{t} j$ cross section increases by about a factor 10, overwhelming the Higgs pair signal. ${ }^{1}$ On the

\footnotetext{
${ }^{1}$ In Ref. [21] we required one lepton with a $p_{T}>10 \mathrm{GeV}$, and one lepton with $p_{T}>15 \mathrm{GeV}$. In this case, the $t \bar{t} j$ background is significantly larger than the signal (see also Ref. [22]). Increasing the lepton transverse momentum cut to $15 \mathrm{GeV}$ solves this problem, while reducing the signal cross section by less than $10 \%$.
}

other hand, if the minimum lepton transverse momentum is increased to $20 \mathrm{GeV}$ which reduces the signal cross section by about $20 \%$, the $t \bar{t} j$ background decreases by one order of magnitude and essentially becomes negligible. However, we emphasize that our matrix element based calculation of the $t \bar{t} j$ background should be viewed with some caution. Effects from hadronization, event pileup and extra jets from initial or final state radiation, as well as detector resolution effects may significantly affect the cross section. For a reliable estimate of the background, a full detector simulation, which is beyond the scope of this paper, is required.

The lepton isolation requirement, together with the lepton $p_{T}$ cut, the $b \rightarrow c \ell \nu$ branching ratio and the di-jet invariant mass cut suppress the $t \bar{t} j$ cross section by about a factor $10^{6}$. A similar suppression factor is also expected in $\ell^{ \pm} \nu b \bar{b}$ $+3 j$ production which also contributes to the background if one of the $b$ quarks decays semileptonically and if the lepton from $b$ decay is isolated. Using the result of Ref. [42], we estimate that the $\ell^{ \pm} \nu b \bar{b}+3 j$ cross section at the LHC is of $\mathcal{O}\left(10^{-3} \mathrm{fb}\right)$ which can safely be neglected.

Our numerical results for the overall normalization of the signal, the $W W W j j, t \bar{t} W$, and the $t \bar{t} t \bar{t}$ background processes agree reasonably well with those reported in Refs. [20] and [22]. For $W Z j j j j$ production, we find a cross section which is about a factor 10 larger. The discrepancy can be traced to the contribution from virtual photon exchange, which was not taken into account in $[20,22]$. No result for $t \bar{t} Z$ production is given in Refs. [20] and [22]. A meaningful comparison of our matrix element based calculation of the $t \bar{t} j$ background and the PYTHIA based estimate in [20,22] is not possible due to the strong dependence of the cross section on the lepton $p_{T}$. 
Overlapping events and double parton scattering are not expected to contribute significantly to the background at the LHC. Contributions from these sources are listed in Table II in the column labeled as "pileup." The numerical values listed were obtained by adding all overlapping event and double parton scattering contributions to the sources of background discussed in Sec. II B, using Eq. (11) and the values of $\mathcal{L}_{b c}$ given in Table I. Since Eq. (11) assumes that that the vertices of the two overlapping events are not resolved, these values are likely conservative. For the SLHC, the pileup cross section in Table II(a) has to be multiplied by a factor 3.7. Our results for the cross section from overlapping events and double parton scattering should be regarded only as order of magnitude estimates. Realistic simulations are needed to draw firm conclusions for this background.

At a $p p$ collider with $\sqrt{s}=200 \mathrm{TeV}$, the cross sections of processes which are dominated by gluon fusion, such as the the $g g \rightarrow H H$ signal, $t \bar{t} t \bar{t}, t \bar{t} Z$ and $t \bar{t} j$ production, are about a factor 100-3000 larger than that at the LHC. Since the cross section for $t \bar{t} t \bar{t}$ production at the LHC is suppressed by the large invariant mass of four top quarks, the increase is particularly large for this process. In contrast, the cross sections of processes dominated by quark-gluon fusion or quark-quark scattering, such as $W W W j j, t \bar{t} W$ and $W W j j j j$ production, increase by only a factor $25-45$. As a result, the $t \bar{t} Z, t \bar{t} j$ and $t \bar{t} t \bar{t}$ backgrounds are relatively more important at the VLHC. The cross sections due to overlapping events and double parton scattering increase by almost three orders of magnitude, and thus may well compete in size with $W W W j j$ production, unless the vertex positions of the overlapping events are resolved. Since the signal is purely gluon induced, the overall signal to background ratio at the VLHC is about a factor 2 better than at the LHC.

All the backgrounds are multi-body production processes, therefore the distribution of the invariant mass, $\sqrt{\hat{s}}$, of the system peaks at values significantly above threshold. In contrast, the signal is a two-body production process for which the $\sqrt{\hat{s}}$ distribution will exhibit a sharper threshold behavior. Unfortunately, with two neutrinos present in the final state, $\sqrt{\hat{s}}$ cannot be reconstructed. However, we anticipate that the invariant mass of all observed final state leptons and jets given by ( $E_{i}$ and $\mathbf{p}_{i}$ are the energies and momenta of the jets and leptons)

$$
m_{v i s}^{2}=\left[\sum_{i=\ell, \ell^{\prime}, \text { jets }} E_{i}\right]^{2}-\left[\sum_{i=\ell, \ell^{\prime}, \mathrm{jets}} \mathbf{p}_{\mathbf{i}}\right]^{2}
$$

will retain most of the expected behavior of the different production processes. Figures 4 (LHC) and 5 (VLHC) clearly demonstrate that this is the case: the signal peaks at smaller values of $m_{v i s}$ than the background processes, especially for lower Higgs boson masses. This distribution, which was not considered in Ref. [20], is what makes possible a $\chi^{2}$ based test to improve extraction of the Higgs boson selfcoupling (see Sec. IV). While detector effects may smear out the tails of this distribution, or shift a peak slightly, it is a
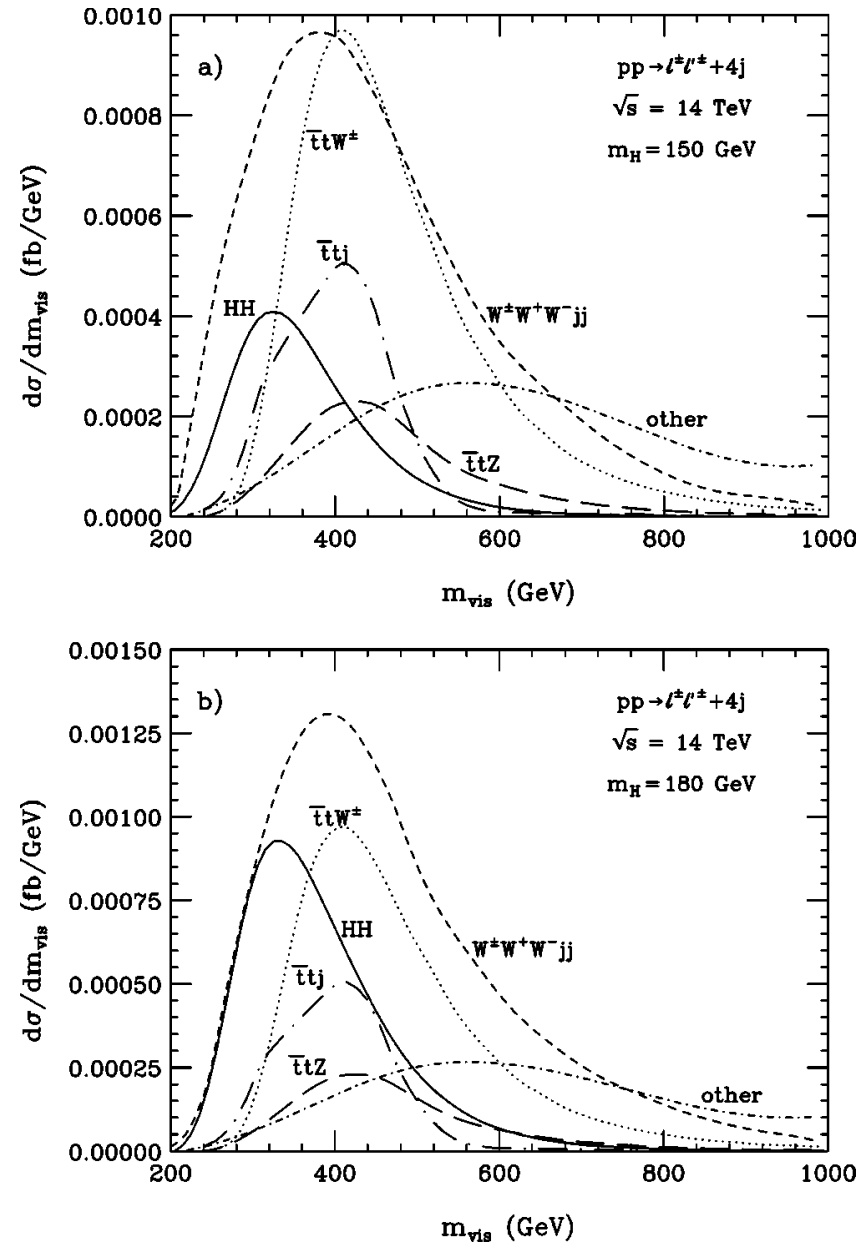

FIG. 4. Distribution of the invariant mass of the observable final state particles, $m_{v i s}$, after all cuts, in $p p \rightarrow \ell^{ \pm} \ell^{\prime \pm}+4 j$ for the signal with (a) $m_{H}=150 \mathrm{GeV}$ and (b) $m_{H}=180 \mathrm{GeV}$, and all backgrounds (except for the contributions from overlapping events and double parton scattering) at the LHC. The dot-dashed curve shows the combined cross section of $W Z j j j j, W W j j j j$ and $t \bar{t} t \bar{t}$ production.

genuine, simple physics effect and care must be taken in any approximations used to simulate the backgrounds that this physics feature is retained.

Since the $W W W j j$ background has a significant contribution from $W H\left(\rightarrow W^{+} W^{-}\right) j j$ production, its $m_{v i s}$ distribution is similar in shape to that of the $H H$ signal. As expected, the $m_{v i s}$ distributions of $t \bar{t} W, t \bar{t} Z$ and $t \bar{t} j$ production peak at similar values, and are similar in shape. The dot-dashed lines in Figs. 4 and 5 represent the combined differential cross section of $W Z j j j j, W W j j j j$ and $t \bar{t} t \bar{t}$ production. It peaks at a much higher visible invariant mass than those of the other background processes. Whereas the signal is concentrated in the region $m_{v i s}<500 \mathrm{GeV}$, the background processes have a significant tail extending to $m_{v i s}=1 \mathrm{TeV}$ and beyond. This makes it possible to normalize the background using data from the $m_{\text {vis }}>500 \mathrm{GeV}$ region. The simple procedure described in Sec. II B 5 for estimating the cross section for overlapping events and double parton scattering is not suitable for calculating distributions. The $m_{v i s}$ distribution of 

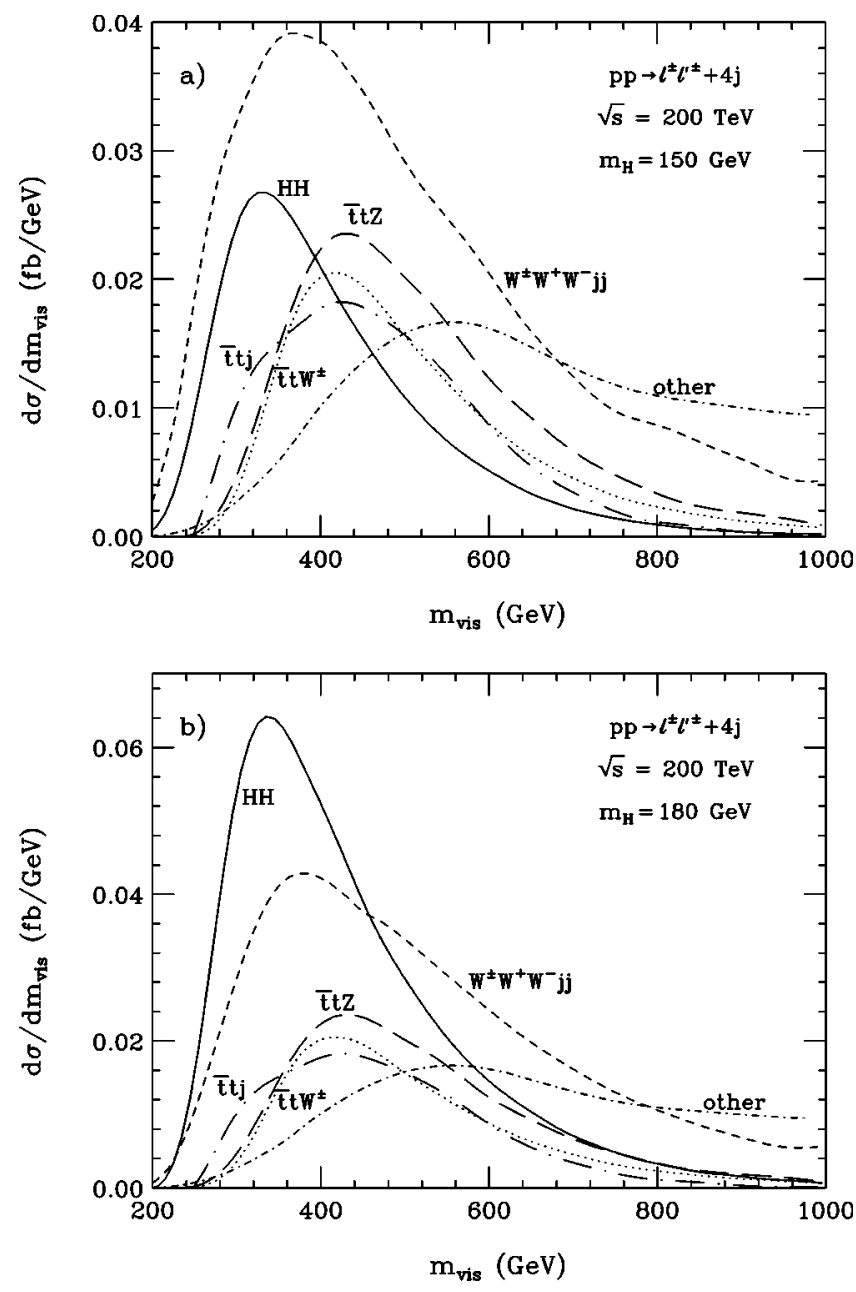

FIG. 5. Distribution of the invariant mass of the observable final state particles, $m_{v i s}$, after all cuts, in $p p \rightarrow \ell^{ \pm} \ell^{\prime \pm}+4 j$ for the signal (solid line) with (a) $m_{H}=150 \mathrm{GeV}$ and (b) $m_{H}=180 \mathrm{GeV}$, and all backgrounds (except for the contributions from overlapping events and double parton scattering) at the VLHC (dashed: $W W W j j$; dotted: $t \bar{t} W$; long-dashed: $t \bar{t} Z$; long-dash-dot: $t \bar{t} j$ ). The dot-dashed curve shows the combined cross section of $W Z j j j j$, $W W j j j j$ and $t \bar{t} t \bar{t}$ production.

these backgrounds therefore is not included in Figs. 4 and 5. With increasing Higgs boson mass, the signal peak gradually moves to higher values of $m_{v i s}$. The efficiency of the $m_{v i s}$ distribution as a discriminator thus decreases somewhat for $m_{H}>180 \mathrm{GeV}$. Comparing the visible invariant mass distributions at the LHC and VLHC, the improved signal to background ratio at the VLHC in the region $m_{v i s}$ $=200-500 \mathrm{GeV}$, where the Higgs pair signal is concentrated, is obvious.

As noted before, all our calculations are consistently performed at leading order, ie. there are precisely four jets (partons) in the final state. In practice, one expects a significant fraction of the $\ell^{ \pm} \ell^{\prime \pm}+4 j$ signal events to contain one or more extra jets originating from initial state gluon radiation. In such events, it is natural to construct $m_{v i s}$ from the four highest $p_{T}$ jets in the event. However, there is no guarantee that the extra jets are always the softest jets in the event.

Since the $m(j j)$ requirement is rather loose [see Eq. (8)], it is conceivable that events where one (or more) of the four jets incorporated in $m_{v i s}$ originate from QCD bremsstrahlung. Hard QCD corrections could also lead to $\ell^{ \pm} \ell^{\prime \pm}+4 j$ events where one of the jets from $W$ decay does not pass the minimum $p_{T}$ cut for jets, but the additional bremsstrahlung jet does. Some of these events might also pass the $m(j j)$ cut. QCD corrections thus could affect the shape of the $m_{v i s}$ distribution. In order to draw firm conclusions, a full calculation of the NLO QCD corrections to $g g \rightarrow H H$ with finite top quark mass effects is needed. Insight may also be gained from performing a calculation where the $g g \rightarrow H H$ matrix elements are interfaced [43] with an event generator such as PYTHIA.

In using PYTHIA for the additional jet radiation, however, one has to be careful. As described previously, the radiation of soft and collinear jets from the initial state is the main source of the large (and top mass independent) QCD corrections to the total signal cross section. The initial state radiation modeled by PYTHIA effectively resums the leading effects of precisely this radiation and includes it in the topology of the final state. Normalizing the rate to the leading order total cross section is therefore inconsistent and the result arbitrary (and not, as often is claimed, a conservative estimate), because the final state topology and the rate are computed in different approximations with a difference which is by no means a reduced higher order uncertainty.

The effect of hard QCD corrections on the $m_{v i s}$ distribution may be reduced by limiting the number of possible $4 j$ combinations which satisfy the cut of Eq. (8). Approximately $60-65 \%(35-40 \%)$ of all signal events have one (two) $4 j$ combination satisfying Eq. (8); almost none have three $4 j$ combinations in the correct invariant mass range. When additional jets are present, many more combinations are possible. Adding the requirement that at most two $4 j$ combinations satisfy Eq. (8) may thus reduce the effect of hard QCD corrections on the $m_{v i s}$ distribution. It may also reduce the signal and background cross sections somewhat. The fraction of events where one or several QCD bremsstrahlung jets pass the cuts may also be reduced by shrinking the $m(j j)$ range in Eq. (8) (see also Ref. [22]). Our choice has been deliberately conservative. Reducing the di-jet invariant mass range to $M_{W} \pm 20 \mathrm{GeV}$ may well be possible [44]. This would also improve the signal to background ratio.

\section{THE THREE LEPTON FINAL STATE}

The calculation of signal and background cross sections for the $\left(j j \ell^{ \pm} \nu\right)\left(\ell^{\prime \pm} \nu \ell^{\prime \prime \mp} \nu\right)$ final state is similar to that described in Sec. II for the same sign di-lepton final state. Due to the smaller branching ratio for leptonic $W$ decays, the cross section is expected to be somewhat smaller than that for the $\left(j j \ell^{ \pm} \nu\right)\left(j j \ell^{\prime \pm} \nu\right)$ channel. The kinematic acceptance cuts for both signal and backgrounds in the $\left(j j \ell^{ \pm} \nu\right)\left(\ell^{\prime \pm} \nu \ell^{\prime \prime \mp} \nu\right)$ final state are

$$
\begin{gathered}
p_{T}(j)>30,20 \mathrm{GeV}, \quad p_{T}(\ell)>15,15,15 \mathrm{GeV}, \\
|\eta(j)|<3.0, \quad|\eta(\ell)|<2.5
\end{gathered}
$$


TABLE III. Higgs pair signal and background cross sections (fb) for $p p \rightarrow \ell^{ \pm} \ell^{\prime \mp} \ell^{\prime \mp}+2 j\left(\ell, \ell^{\prime}=e, \mu, \ell \neq \ell^{\prime}\right)$ at the VLHC $(\sqrt{s}=200 \mathrm{TeV})$, imposing the cuts listed in Eqs. (14)-(16), and as a function of the Higgs boson mass $(\mathrm{GeV})$. The background labeled "pileup" represents a rough estimate of the combined $W W W j j$, $t \bar{t} W, t \bar{t} Z$, and $t \bar{t} t \bar{t}$ cross section from overlapping events and double parton scattering. The last column, labeled $\mathcal{B}_{\text {tot }}$, shows the total background cross section.

\begin{tabular}{ccccccccc}
\hline \hline$m_{H}$ & $H H$ & $W W W j j$ & $t \bar{t} W$ & $t \bar{t} Z$ & $t \bar{t} j$ & $t \bar{t} t \bar{t}$ & pileup & $\mathcal{B}_{\text {tot }}$ \\
\hline 150 & 1.40 & 1.43 & 0.39 & 1.39 & 0.45 & 0.47 & $\sim 2.4$ & 6.53 \\
160 & 3.06 & 1.96 & 0.39 & 1.39 & 0.45 & 0.47 & $\sim 2.4$ & 7.06 \\
180 & 3.04 & 1.71 & 0.39 & 1.39 & 0.45 & 0.47 & $\sim 2.4$ & 6.81 \\
200 & 1.66 & 1.47 & 0.39 & 1.39 & 0.45 & 0.47 & $\sim 2.4$ & 6.57 \\
\hline \hline
\end{tabular}

$$
\Delta R(j j)>0.6, \quad \Delta R(j \ell)>0.4, \quad \Delta R(\ell \ell)>0.2 .
$$

In addition, we impose the di-jet invariant mass cut of Eq. (8).

Except for $W^{ \pm} W^{ \pm} j j j j$ and $W^{ \pm} Z j j j j$ production, all processes discussed in Sec. II B contribute to the background. For final states containing a same flavor opposite sign lepton pair, $W^{ \pm} \ell^{+} \ell^{-} j j$ production constitutes an additional source of background. We have calculated the $W^{ \pm} \ell^{+} \ell^{-} j j$ background using the exact matrix elements of Ref. [37]. The cross section for $W^{ \pm} \ell^{+} \ell^{-} j j$ production is about three orders of magnitude larger than the Higgs boson pair signal, if photon exchange diagrams are taken into account. To increase the signal to background ratio, one can either impose a minimum $\ell^{+} \ell^{-}$invariant mass cut, or increase the $\Delta R\left(\ell^{+} \ell^{-}\right)$cut. Unfortunately, due to correlations between the momenta of the fermions in the $H \rightarrow W^{+} W^{-} \rightarrow 4$ fermion decay [29], the $\ell^{+} \ell^{-}$invariant mass tends to be rather small in Higgs pair events. As a result, the signal to background ratio cannot be improved to better than 1:100 without reducing the signal cross section to an unacceptably low level.

In the following we therefore only consider $\ell^{ \pm} \ell^{\prime \mp} \ell^{\prime \mp}$ $+2 j, \ell \neq \ell^{\prime}$, production. Excluding all final states containing a same flavor opposite sign lepton pair reduces the signal cross section by a factor 4 . As a result, the cross section at the LHC becomes too small to be of interest; for an integrated luminosity of $300 \mathrm{fb}^{-1}$ only 8 events are expected. We therefore present numerical results at VLHC energies only in this section.

The total cross sections within cuts [see Eqs. (14)-(16)] for signal and background processes at the VLHC are listed in Table III. The signal cross section is about a factor 5 smaller than in the same-sign dilepton case. The loss in signal however is at least partially compensated by the significantly improved signal to background ratio. This becomes more evident in Fig. 6 which shows the $m_{v i s}$ distribution for $m_{H}=150 \mathrm{GeV}$ and $m_{H}=180 \mathrm{GeV}$. Here, $m_{v i s}$ is defined by Eq. (13), using the four-momentum vectors of the three charged leptons and the two jets. The largest contribution to the background in the region where the signal peaks comes from $t \bar{t} Z$ production. While the $t \bar{t} j$ and $t \bar{t} t \bar{t}$ total cross sections are similar (see Table III), the $t \bar{t} t \bar{t}$ contribution to the
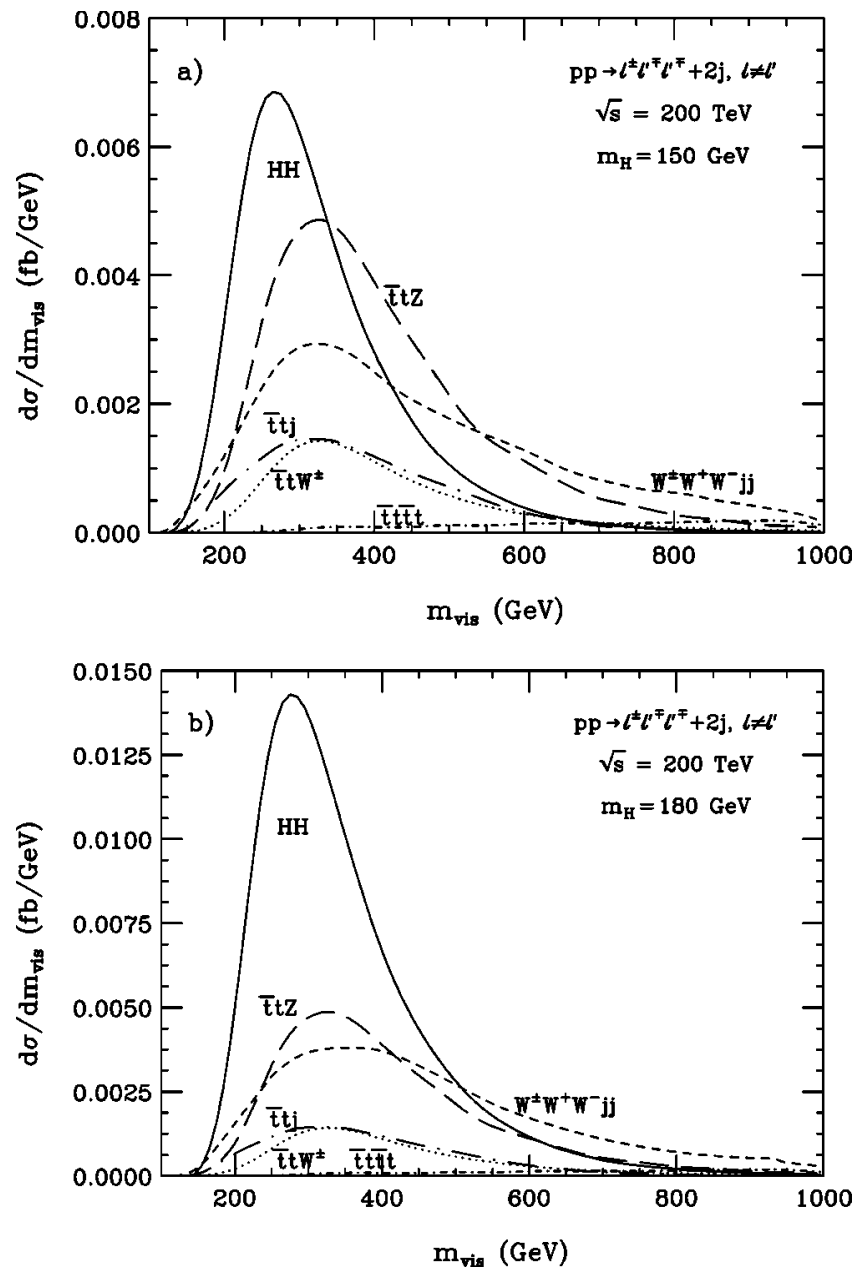

FIG. 6. Distribution of the invariant mass of the observable final state particles, $m_{v i s}$, after all cuts, in $p p \rightarrow \ell^{ \pm} \ell^{\prime \mp} \ell^{\prime \mp}+2 j, \ell$ $\neq \ell^{\prime}$, for the signal (solid line) with (a) $m_{H}=150 \mathrm{GeV}$ and (b) $m_{H}=180 \mathrm{GeV}$, and all backgrounds (except for the contributions from overlapping events and double parton scattering) at the VLHC (dashed: $W W W j j$; dotted: $t \bar{t} W$; long-dashed: $t \bar{t} Z$; long-dash-dot: $t \bar{t} j$; dot-dashed: $t \bar{t} t \bar{t}$ ).

background in this region is negligible. Most $t \bar{t} t \bar{t}$ events have visible invariant masses well in excess of $1 \mathrm{TeV}$. The signal to background ratio for the $\ell^{ \pm} \ell^{\prime \mp} \ell^{\prime \mp}+2 j$ channel is approximately a factor two better than for the same-sign dilepton final state (see Fig. 5).

Since there are fewer jets present, initial state gluon radiation should have a smaller effect on the $m_{v i s}$ distribution in the three lepton final state than in the same-sign dilepton case.

\section{DETERMINING THE HIGGS BOSON SELF-COUPLING}

The Feynman diagrams contributing to $g g \rightarrow H H$ in the SM consist of fermion triangle and box diagrams [16]. Nonstandard Higgs boson self-couplings affect only the triangle diagrams with a Higgs boson exchanged in the $s$ channel. As this is a two-body production process, the self-coupling con- 

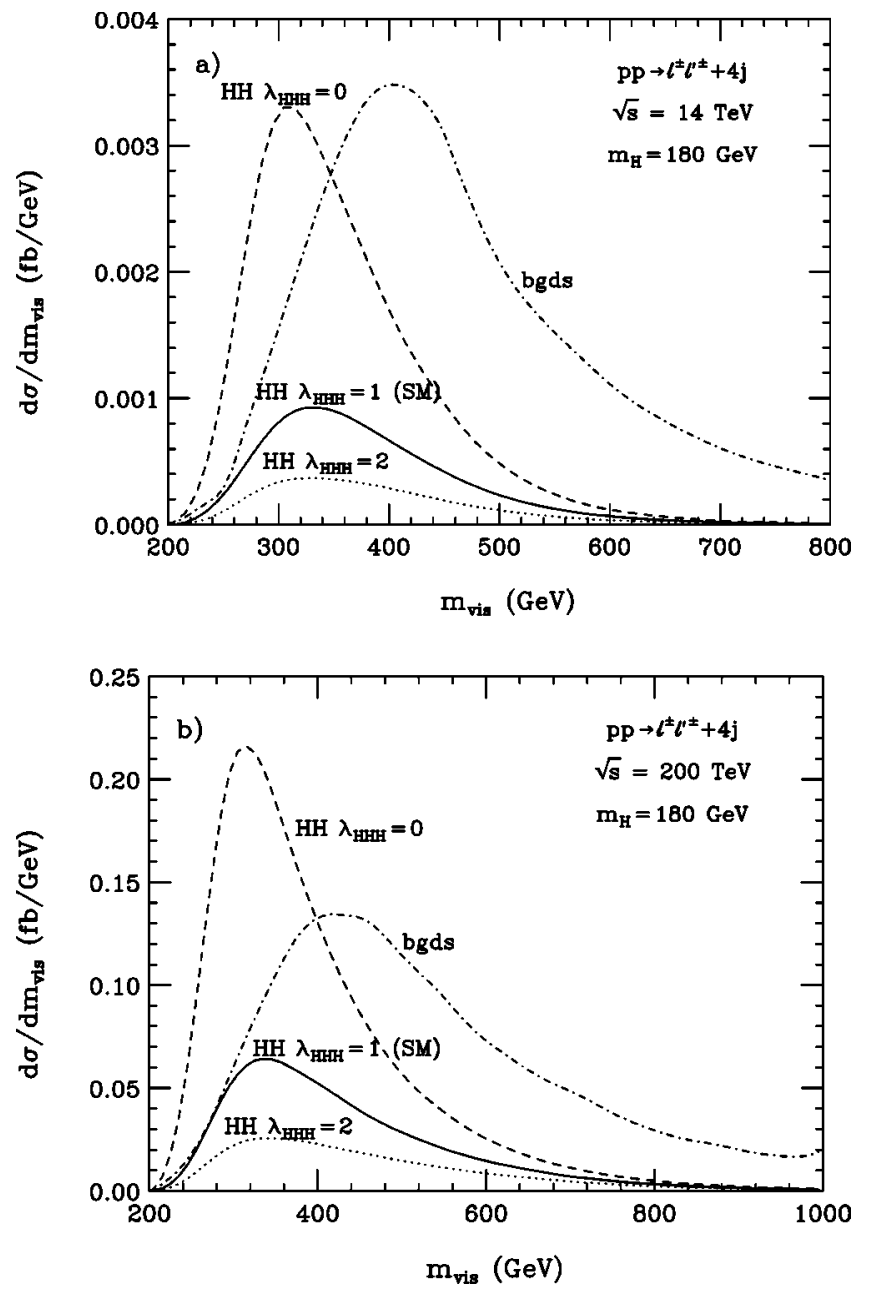

FIG. 7. The $m_{v i s}$ distribution of the signal for $p p \rightarrow \ell^{ \pm} \ell^{\prime \pm}$ $+4 j$ and $m_{H}=180 \mathrm{GeV}$ at (a) the LHC, and (b) the VLHC, in the SM (solid curve), for $\lambda_{H H H}=\lambda / \lambda_{S M}=0$ (dashed line) and for $\lambda_{H H H}=2$ (dotted line). The dot-dashed line shows the combined $m_{v i s}$ distribution of all background processes (except those from overlapping events and double parton scattering). Qualitatively similar results are obtained for other values of $m_{H}$.

tribution will affect lower values of $m_{v i s}$. The box contribution, however, contains a final state momentum dependence which modifies the typical two-body threshold dependence. Thus a change in $\lambda$ will lead to a shape change of the signal $m_{v i s}$ distribution. This is illustrated in Fig. 7 for the $\ell^{ \pm} \ell^{\prime \pm}$ $+4 j$ final state with $m_{H}=180 \mathrm{GeV}$ and two non-standard values of $\lambda_{H H H}=\lambda / \lambda_{S M}$. Since box and triangle diagrams interfere destructively, the $g g \rightarrow H H$ cross section for 1 $<\lambda_{H H H}<2.7$ is smaller than in the SM. The absence of a Higgs boson self-coupling $\left(\lambda_{H H H}=0\right)$ results in a Higgs pair production cross section which is about a factor 3 larger than the SM result. Figure 7 also demonstrates that the $m_{v i s}$ distribution of the signal peaks at a smaller value than that of the combined background. This remains true for other Higgs boson masses, as long as $m_{H} \leqslant 200 \mathrm{GeV}$.

The shape change of the $m_{v i s}$ distribution induced by nonstandard values of $\lambda_{H H H}$ can be used to derive quantitative sensitivity bounds on the Higgs boson self-coupling. We accomplish this by calculating $95 \%$ confidence level (C.L.) limits performing a $\chi^{2}$ test. The statistical significance is calculated by splitting the $m_{v i s}$ distribution into a number of bins, each with typically more than five events. In each bin the Poisson statistics are approximated by a Gaussian distribution. We impose the cuts described in Secs. II and III and combine channels with electrons and muons in the final state, conservatively assuming a common lepton identification efficiency of $\epsilon=0.85$ for each lepton. Except for the Higgs boson self-coupling we assume the SM to valid: by the time a measurement of $\lambda$ is attempted, the Higgs boson mass will be precisely known, and the $t \bar{t} H$ coupling and the $H$ $\rightarrow W^{+} W^{-}$branching ratio will have been measured with a precision of $15 \%$ or better at the LHC and/or an $e^{+} e^{-}$linear collider $[9,45]$. We include all background processes listed in Table II and Table III, except those from overlapping events and double parton scattering. The challenge of including higher order effects is considerably more complicated for the background than for the $H H$ signal, where at least the physics interpretation is clear as previously discussed. The aim for the backgrounds is not to capture the bulk of events after cuts. Instead, one tries to cut into the tails of distributions, where the impact of higher order corrections might be very different. Therefore an analysis should depend as little as possible on the background rates [46], while a dependence on the signal rate is unfortunately unavoidable for any new physics process, which by definition will rely on comparably fewer, rare events. To show that our analysis satisfies this requirement, and approximately take into account the unknown NLO QCD effects, we perform two separate calculations of sensitivity limits:

(1) we assume a uniform $K$ factor of $K=1$ for the $m_{v i s}$ distribution of the background but allow for a normalization uncertainty of $\Delta \mathcal{N}=30 \%$ of the SM cross section;

(2) we assume a uniform $K$ factor of $K=1.3$ for the $m_{v i s}$ distribution of the background and allow for a normalization uncertainty of $\Delta \mathcal{N}=10 \%$ of the SM cross section.

The results from both calculations are then compared and the more conservative bound is selected. Since the background cross section can be directly determined from the high $m_{v i s}$ region with a statistical precision of $15 \%$ or better for the assumed integrated luminosities, the bounds we derive should be conservative.

The expression for $\chi^{2}$ which we use to compute confidence levels is given by [47]

$$
\chi^{2}=\sum_{i=1}^{n_{D}} \frac{\left(N_{i}-f N_{i}^{0}\right)^{2}}{f N_{i}^{0}}+\left(n_{D}-1\right)
$$

where $n_{D}$ is the number of bins, $N_{i}$ is the number of events for a given $\Delta \lambda_{H H H}=\left(\lambda-\lambda_{S M}\right) / \lambda_{S M}$, and $N_{i}^{0}$ is the number of events in the SM in the $i$ th bin. $f$ reflects the uncertainty in the normalization of the SM cross section within the allowed range, and is determined by minimizing $\chi^{2}$ :

$$
f= \begin{cases}(1+\Delta \mathcal{N})^{-1} & \text { for } \bar{f}<(1+\Delta \mathcal{N})^{-1} \\ \bar{f} & \text { for }(1+\Delta \mathcal{N})^{-1}<\bar{f}<1+\Delta \mathcal{N}, \\ 1+\Delta \mathcal{N} & \text { for } \bar{f}>1+\Delta \mathcal{N},\end{cases}
$$




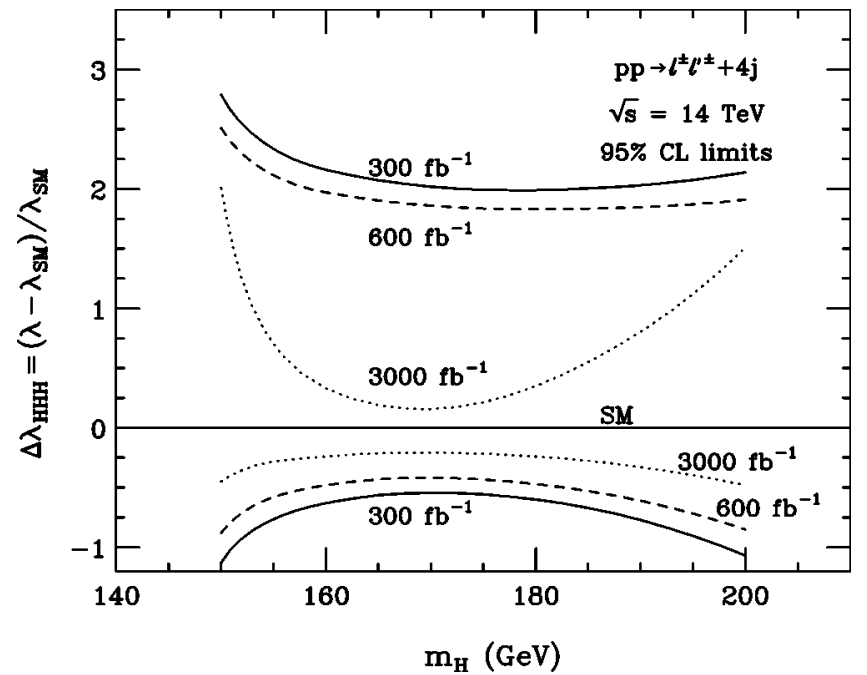

FIG. 8. Limits achievable at $95 \%$ C.L. for $\Delta \lambda_{H H H}=(\lambda$ $\left.-\lambda_{S M}\right) / \lambda_{S M}$ in $p p \rightarrow \ell^{ \pm} \ell^{\prime \pm}+4 j$ at the LHC. Bounds are shown for integrated luminosities of $300 \mathrm{fb}^{-1}$ (solid lines), $600 \mathrm{fb}^{-1}$ (dashed lines) and $3000 \mathrm{fb}^{-1}$ (dotted lines). The allowed region is between the two lines of equal texture. The Higgs boson selfcoupling vanishes for $\Delta \lambda_{H H H}=-1$.

with

$$
\bar{f}^{2}=\left\{\sum_{i=1}^{n_{D}} N_{i}^{0}\right\}^{-1} \sum_{i=1}^{n_{D}} \frac{N_{i}^{2}}{N_{i}^{0}}
$$

For the LHC, we derive sensitivity limits for integrated luminosities of $300 \mathrm{fb}^{-1}, 600 \mathrm{fb}^{-1}$ and $3000 \mathrm{fb}^{-1}$, and Higgs boson masses in the range $150 \mathrm{GeV} \leqslant m_{H}$ $\leqslant 200 \mathrm{GeV}$. An integrated luminosity of $300 \mathrm{fb}^{-1}$ $\left(600 \mathrm{fb}^{-1}\right)$ corresponds to 3 years of running at the LHC design luminosity with one (two) detectors. The larger value of $3000 \mathrm{fb}^{-1}$ can be achieved in about 3 years of running at the SLHC with one detector. Since the cross section for Higgs boson pair production in the three lepton final state is very small, we calculate sensitivity bounds only for the same-sign dilepton channel. Our results are shown in Fig. 8, which demonstrates that, for $300 \mathrm{fb}^{-1}$, a vanishing Higgs boson self-coupling $\left(\Delta \lambda_{H H H}=-1\right)$ is excluded at the $95 \%$ C.L. or better, and that $\lambda$ can be determined with a precision of up to $-60 \%$ and $+200 \%$. Doubling the integrated luminosity to $600 \mathrm{fb}^{-1}$ improves the sensitivity by $10-25 \%$. For $300 \mathrm{fb}^{-1}$ and $600 \mathrm{fb}^{-1}$, the bounds for positive values of $\Delta \lambda_{H H H}$ are significantly weaker than those for $\Delta \lambda_{H H H}<0$, due to the limited number of signal events in this region of parameter space. At the SLHC, for $3000 \mathrm{fb}^{-1}$, the Higgs boson self-coupling can be determined with an accuracy of $20-30 \%$ for $160 \mathrm{GeV} \leqslant m_{H} \leqslant 180 \mathrm{GeV}$. The significance of the SM signal for $300 \mathrm{fb}^{-1}\left(3000 \mathrm{fb}^{-1}\right)$ is slightly more than $1 \sigma(3 \sigma)$ for $m_{H}=150 \mathrm{GeV}$ and $200 \mathrm{GeV}$, and about $2.5 \sigma(10 \sigma)$ for Higgs boson masses between $160 \mathrm{GeV}$ and $180 \mathrm{GeV}$. The results shown in Fig. 8 are about $5-10 \%$ weaker than those found in Ref. [21] where only the dominant $W W W j j$ and $t \bar{t} W$ backgrounds where taken into account while the effect of all other backgrounds was simu-
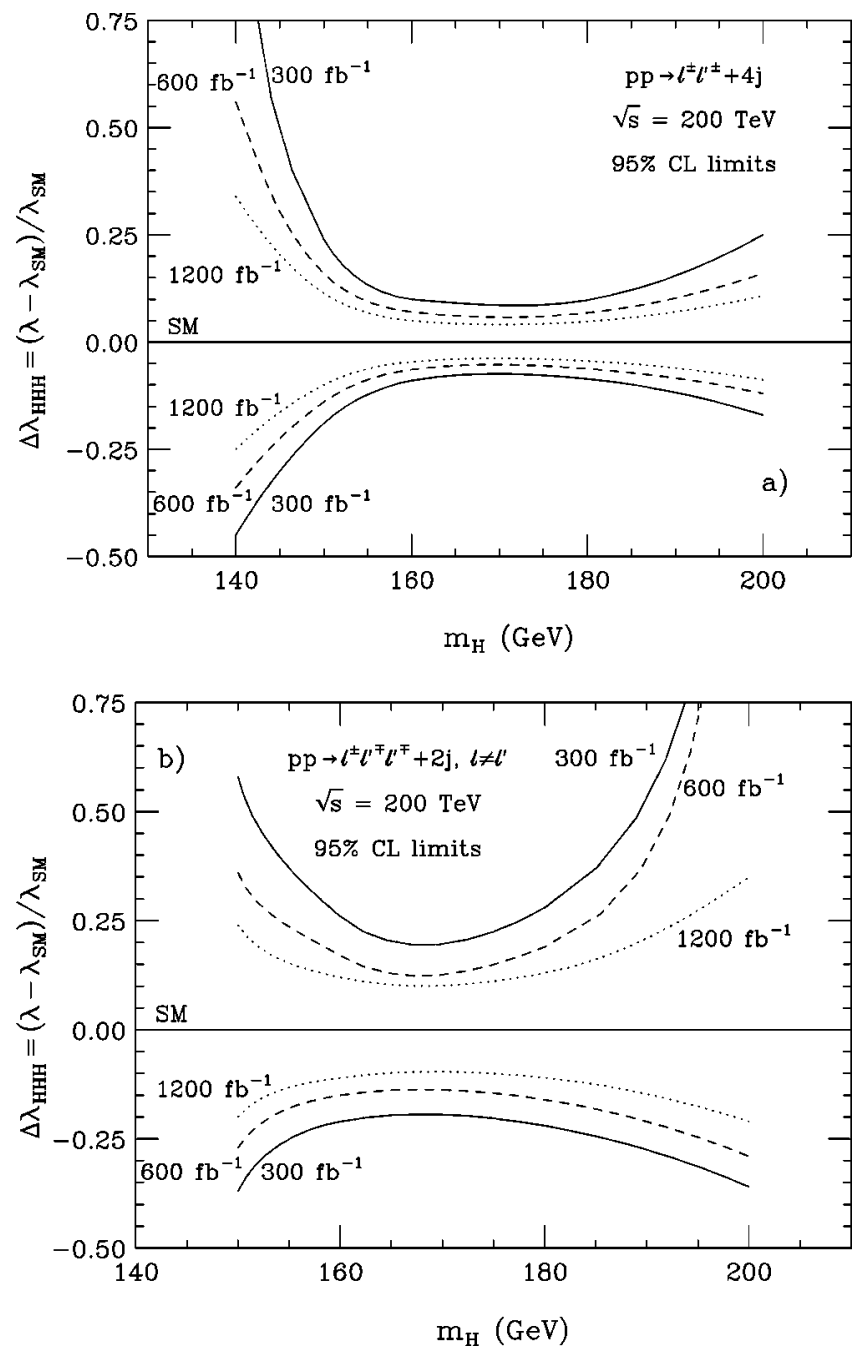

FIG. 9. Limits achievable at $95 \%$ C.L. for $\Delta \lambda_{H H H}=(\lambda$ $\left.-\lambda_{S M}\right) / \lambda_{S M}$ for (a) $p p \rightarrow \ell^{ \pm} \ell^{\prime \pm}+4 j$ and (b) $p p \rightarrow \ell^{ \pm} \ell^{\prime \mp} \ell^{\prime \mp}$ $+2 j$ at the VLHC. Bounds are shown for integrated luminosities of $300 \mathrm{fb}^{-1}$ (solid lines), $600 \mathrm{fb}^{-1}$ (dashed lines) and $1200 \mathrm{fb}^{-1}$ (dotted lines). The allowed region is between the two lines of equal texture. The Higgs boson self-coupling vanishes for $\Delta \lambda_{H H H}=-1$.

lated by multiplying the combined $W W W j j$ and $t \bar{t} W$ visible invariant mass distribution by a factor 1.1 .

For the VLHC, we calculate bounds for both the $\ell^{ \pm} \ell^{\prime \pm}$ $+4 j$ and the $\ell^{ \pm} \ell^{\prime \mp} \ell^{\prime \mp}+2 j$ final states. We assume integrated luminosities of $300 \mathrm{fb}^{-1}, 600 \mathrm{fb}^{-1}$ and $1200 \mathrm{fb}^{-1}$. For a design luminosity of $\mathcal{L}=2 \times 10^{34} \mathrm{~cm}^{-2} \mathrm{~s}^{-1}$ [24], the latter corresponds to 3 years of running with two detectors. The $95 \%$ C.L. limits which one may hope to achieve at such a machine are shown in Fig. 9.

At a $p p$ collider with $\sqrt{s}=200 \mathrm{TeV}$ and an integrated luminosity of $300 \mathrm{fb}^{-1}$, the Higgs boson self-coupling can be measured with a precision of $8-25 \%$ at $95 \%$ C.L. for $150 \mathrm{GeV}<m_{H}<200 \mathrm{GeV}$. For $1200 \mathrm{fb}^{-1}$, the bounds improve to $4-11 \%$. Although the signal to background ratio in the $\ell^{ \pm} \ell^{\prime \mp} \ell^{\prime \mp}+2 j$ channel is significantly better, the sensitivity limits which can be achieved are about a factor 2 to 3 weaker than those obtained for the $\ell^{ \pm} \ell^{\prime \pm}+4 j$ final state, 
due to the reduced number of signal events.

Our calculation of sensitivity bounds for $\lambda$ is subject to several uncertainties which should be addressed in a future more detailed analysis. In calculating limits, we have ignored the background from overlapping events and double parton scattering. Their sizes depend sensitively on the accelerator parameters, in particular the bunch spacing, and on the ability of the detectors to resolve the vertices of such events. In addition, these types of background are difficult to model at the parton level. Our estimates (see Sec. II B 5) indicate that they are small at the LHC, but may not be negligible at the SLHC or VLHC. A conservative upper limit of how much the background from overlapping events and double parton scattering may change the bounds on $\lambda$ is obtained by assuming that the $m_{v i s}$ distribution of such events peaks in the region where also the signal reaches its maximum. Assuming that this is the case, and using the results of Table II, one finds that the SLHC (VLHC) limits weaken by at most $5 \%$ $(15 \%)$.

We also ignored the contributions from $W W Z j j$ and $W^{+} W^{-} W^{+} W^{-}$production in our calculation. The cross section of these processes is small compared to that of the dominant background contributions. They therefore should have a negligible effect on the bounds which can be obtained. The extremely large number of Feynman diagrams contributing to $W Z j j j j$ and $W W j j j j$ production makes a calculation employing exact matrix elements currently impractical. To calculate the cross section for these processes we interfaced the $p p \rightarrow W Z j j$ and $p p \rightarrow W W j j$ matrix elements with PYTHIA. This procedure may well result in cross sections which differ from the correct result by a factor $1.5^{ \pm 1}$. However, both the $W Z j j j j$ and the $W W j j j j$ cross sections are small in the region of $m_{v i s}$ where the signal distribution peaks. Uncertainties in the calculation of their production cross sections thus should not change the bounds on $\lambda$ by more than a few percent. To substantiate this claim, we have varied the $W Z j j j j$ cross section by a factor $2^{ \pm 1}$ and recomputed the $95 \%$ C.L. limits for $\lambda$. The values obtained differ from those shown in Figs. 8 and 9 by at most $5 \%$.

Uncertainties in the extraction of sensitivity limits for $\lambda$ also arise from the $t \bar{t} j$ background which we calculated at the parton level. Since the cross section of the $t \bar{t} j$ background sensitively depends on the lepton $p_{T}$ cut and also the lepton isolation requirement, detector resolution effects may have a significant effect. Varying the $t \bar{t} j$ cross section by a factor $1.5^{ \pm 1}$ changes the limits for $\lambda$ by about $5-10 \%$. Finally, QCD corrections are expected to modify the shape of the $m_{v i s}$ distribution for both signal and background. In our calculation we have approximated the effect of QCD corrections by uniform $K$ factors which do not take into account this effect. While an accurate answer how QCD corrections affect the shape of the $m_{v i s}$ distribution requires the calculation of next-to-leading corrections to signal and background processes, it seems unlikely that they will change the sensitivity bounds by more than $20 \%$.

Our calculation of sensitivity bounds for $\lambda$ has been based on a simple $\chi^{2}$ test of the $m_{v i s}$ distribution. More powerful statistical tools, or a neural net analysis, may considerably improve the limits which can be achieved.

\section{DISCUSSION AND CONCLUSIONS}

A direct experimental investigation of the Higgs potential represents a conclusive test of the mechanism of electroweak symmetry breaking and mass generation. After the discovery of an elementary Higgs boson and the test of its couplings to fermions and gauge bosons, experimental evidence that the shape of the Higgs potential has the form required for breaking the electroweak symmetry will complete the proof that the masses of fermions and weak bosons are generated by spontaneous symmetry breaking. In order to probe the shape of the Higgs potential, the Higgs boson self-coupling must be determined.

The Higgs boson self-coupling can be measured in Higgs boson pair production at lepton or hadron colliders. In this paper, we presented a detailed analysis of Higgs boson pair production via gluon fusion with subsequent decay into four $W$ bosons at the LHC, a luminosity upgraded LHC (SLHC), and a planned next-generation hadron collider with a center of mass energy of $\sqrt{s}=200 \mathrm{TeV}$ (VLHC). We considered two final states: $\ell^{ \pm} \ell^{\prime \pm}+4$ jets and $\ell^{ \pm} \ell^{\prime \mp} \ell^{\prime \mp}+2$ jets. To calculate the signal cross section, exact one-loop matrix elements for finite top quark masses were used. Final state spin correlations for the $H \rightarrow W W \rightarrow 4$ fermion decay were fully taken into account, together with finite $W$ and Higgs boson width effects.

We investigated in detail which processes contribute to the background, including backgrounds from overlapping events and double parton scattering. All background cross sections, except those for $W W j j j j$ and $W Z j j j j$ production, were calculated using exact tree level matrix elements. Contributions to the background from overlapping events depend on the ability of detectors to resolve vertex positions, and on machine parameters. We presented a simple order-ofmagnitude estimate of the cross section from overlapping events which indicates that these should not be a problem at the LHC. At the SLHC and VLHC, however, the background from overlapping events could be non-negligible.

At the LHC, the total background cross section is significantly larger than that of the signal in the $\ell^{ \pm} \ell^{\prime \pm}+4 j$ channel. There are too few events in the $\ell^{ \pm} \ell^{\prime \mp} \ell^{\prime \mp}+2 j$ channel to make it useful. However, the distribution of the visible invariant mass of the final state particles, $m_{v i s}$, for most of the processes contributing to the background peaks at a considerably higher value of $m_{v i s}$ than that of the signal, regardless of the value of $\lambda$. The shape of the $m_{v i s}$ distribution can thus be used as a tool to derive limits on the Higgs boson self-coupling, $\lambda$.

At the VLHC, we found an improved signal to background ratio for the $\ell^{ \pm} \ell^{\prime \pm}+4 j$ channel. The $\ell^{ \pm} \ell^{\prime \mp} \ell^{\prime \mp}$ $+2 j$ final state has an even more advantageous signal to background ratio, however, the signal cross section is significantly smaller than that for $p p \rightarrow H H \rightarrow \ell^{ \pm} \ell^{\prime \pm}+4 j$.

In order to determine how well one can hope to measure the Higgs boson self-coupling at future hadron colliders, we have performed a $\chi^{2}$ test of the $m_{v i s}$ distribution. We found 
that, at the LHC, with $300 \mathrm{fb}^{-1}$, one will be able to perform a first, albeit not very precise, measurement of the Higgs boson self-coupling. The non-vanishing of $\lambda$, however, can be established at $95 \%$ C.L. or better for $150 \mathrm{GeV}<m_{H}$ $<200 \mathrm{GeV}$. This alone is an important, non-trivial test of spontaneous symmetry breaking; the exact non-zero value of $\lambda$ may vary depending on the way nature chooses to spontaneously break the electroweak symmetry. At the SLHC, for $3000 \mathrm{fb}^{-1}$, a measurement with a precision of up to $20 \%$ at 95\% C.L. is possible; $\lambda$ at the SLHC can be determined with an accuracy of $10-30 \%$ at the $1 \sigma$ level for Higgs boson masses between 150 and $200 \mathrm{GeV}$. Compared with an estimate based on the total cross section [22], the fit to the $m_{v i s}$ distribution improves the accuracy of the measurement of Higgs self-coupling by a factor 1.2 to 2.5 . For the same range of $m_{H}$, the $95 \%$ C.L. bounds on $\lambda$ for a $200 \mathrm{TeV} p p$ collider (see Fig. 9) indicate that deviations of $10 \%$ or less from the SM value of $\lambda$ can be measured at 95\% C.L. if more than $1 \mathrm{ab}^{-1}$ can been accumulated. Because of the reduced signal rate, limits obtained from the $\ell^{ \pm} \ell^{\prime \mp} \ell^{\prime \mp}$ $+2 j$ final state are about a factor 2 to 3 weaker than those extracted from the $\ell^{ \pm} \ell^{\prime \pm}+4 j$ channel.

It is interesting to compare the sensitivities which one may hope to achieve at the LHC, SLHC and VLHC with those obtained for future $e^{+} e^{-}$linear colliders [15,17-19]. At DESY TeV Energy Superconducting Linear Accelerator (TESLA) energies, $\sqrt{s}=500-800 \mathrm{GeV}$, the Higgs boson self-coupling can only be determined if $m_{H}<140 \mathrm{GeV}$. For larger values of $m_{H}$, the cross section for the dominant Higgs pair production process, $e^{+} e^{-} \rightarrow \mathrm{ZHH}$, is too small for a useful measurement. For $m_{H}=120 \mathrm{GeV}, \quad \sqrt{s}$ $=500 \mathrm{GeV}$, and $1 \mathrm{ab}^{-1}$, one finds that $\lambda$ can be measured with a precision of $\delta \lambda= \pm 0.20$ for one sigma [18]. In contrast, Higgs boson pair production followed by decays into four $W$ bosons at the LHC and SLHC offers an opportunity to probe the Higgs boson self-coupling for masses in the range $150 \mathrm{GeV}<m_{H}<200 \mathrm{GeV}$. For $m_{H}<140 \mathrm{GeV}$, where the decay $H \rightarrow b \bar{b}$ dominates, the QCD $b \bar{b} b \bar{b}$ background is so large that a measurement of the Higgs boson self-coupling is hopeless. LHC and a linear collider operating in the range of $\sqrt{s}=500-1000 \mathrm{GeV}$ thus complement each other in their abilities to determine $\lambda$.

A more direct comparison can be carried out between the VLHC and CLIC, a proposed multi-TeV $e^{+} e^{-}$linear collider [48]. For $m_{H}=180 \mathrm{GeV}$, one finds [15] that, for $e^{+} e^{-}$collisions at $\sqrt{s}=3 \mathrm{TeV}$ and an integrated luminosity of $5 \mathrm{ab}^{-1}, \lambda$ can be determined with a precision of $\delta \lambda=$ $\pm 0.080(1 \sigma)$. For the same Higgs boson mass, the Higgs boson self-coupling can be measured with an accuracy of $\delta \lambda= \pm 0.035$ at a $200 \mathrm{TeV} p p$ collider with $300 \mathrm{fb}^{-1}$.

Our analysis has been based on leading order parton level calculations. This introduces uncertainties in our derivation of sensitivity bounds which we estimated to be of $\mathcal{O}(20 \%)$. In order to derive more realistic limits for the Higgs boson self-coupling, more detailed simulations which take into account detector effects, as well as the effects of higher order QCD corrections are needed.

\section{ACKNOWLEDGMENTS}

We would like to thank K. Desch, S. Dittmaier, I. Hinchliffe, K. Jakobs, F. Mazzucato, S. Mrenna, F. Piccinini, M. Spira, T. Stelzer, D. Zeppenfeld and P.M. Zerwas for useful discussions. One of us (U.B.) would like to thank the Phenomenology Institute of the University of Wisconsin, Madison, and the Fermilab Theory Group, where part of this work was carried out, for their generous hospitality and for financial support. This research was supported in part by the University of Wisconsin Research Committee with funds granted by the Wisconsin Alumni Research Foundation, by the U. S. Department of Energy under Contracts No. DE-FG0295ER40896 and No. DE-AC02-76CH03000, and the National Science Foundation under grants No. PHY-9970703 and No. PHY-0139953.
[1] D. Abbaneo et al., (LEPEWWG), hep-ex/0212036; M. Grünewald, to appear in the Proceedings of the 31st International Conference on High Energy Physics, Amsterdam, The Netherlands, 2002, hep-ex/0210003.

[2] M. Dittmar and H.K. Dreiner, Phys. Rev. D 55, 167 (1997); D. Rainwater and D. Zeppenfeld, ibid. 60, 113004 (1999); 61, 099901(E) (2000); N. Kauer, T. Plehn, D. Rainwater, and D. Zeppenfeld, Phys. Lett. B 503, 113 (2001); and for an experimental perspective, see e.g. K. Jakobs, "Search for the Standard Model Higgs boson using vector boson fusion," talk presented at ATLAS week, Clermont-Ferrand, 2002.

[3] Z. Kunszt and F. Zwirner, Nucl. Phys. B385, 3 (1992); ATLAS TDR, report CERN/LHCC/99-15, 1999; CMS TP, report CERN/LHCC/94-38, 1994; M. Spira, Fortschr. Phys. 46, 203 (1998); M. Spira, A. Djouadi, D. Graudenz, and P.M. Zerwas, Nucl. Phys. B453, 17 (1995); M. Krämer, E. Laenen, and M. Spira, ibid. B511, 523 (1998); S. Catani, D. de Florian, and M. Grazzini, J. High Energy Phys. 05, 025 (2001); R.V. Harlander and W.B. Kilgore, Phys. Rev. D 64, 013015 (2001); C. Anastasiou and K. Melnikov, Nucl. Phys. B646, 220 (2002).

[4] D. Rainwater, D. Zeppenfeld, and K. Hagiwara, Phys. Rev. D 59, 014037 (1999); T. Plehn, D. Rainwater, and D. Zeppenfeld, Phys. Lett. B 454, 297 (1999); Phys. Rev. D 61, 093005 (2000).

[5] O.J. Éboli and D. Zeppenfeld, Phys. Lett. B 495, 147 (2000).

[6] T. Plehn and D. Rainwater, Phys. Lett. B 520, 108 (2001); T. Han and B. McElrath, ibid. 528, 81 (2002).

[7] D. Zeppenfeld, R. Kinnunen, A. Nikitenko, and E. RichterWas, Phys. Rev. D 62, 013009 (2000).

[8] V. Drollinger, T. Müller, and D. Denegri, hep-ph/0201249.

[9] F. Maltoni, D. Rainwater, and S. Willenbrock, Phys. Rev. D 66, 034022 (2002).

[10] D. Zeppenfeld, in Proceedings of the APS/DPF/DPB Summer Study on the Future of Particle Physics (Snowmass 2001), edited by R. Davidson and C. Quigg, hep-ph/0203123; F. Piccinini, to appear in the Proceedings of the 31st International 
Conference on High Energy Physics, Amsterdam, The Netherlands, 2002 hep-ph/0209377; A. Belyaev and L. Reina, J. High Energy Phys. 08, 041 (2002).

[11] ECFA/DESY LC Physics Working Group Collaboration, J.A. Aguilar-Saavedra et al., hep-ph/0106315, and references therein; American Linear Collider Working Group Collaboration, T. Abe et al., in Proceedings of the APS/DPF/DPB Summer Study on the Future of Particle Physics (Snowmass 2001), edited by R. Davidson and C. Quigg, hep-ex/0106056, and references therein.

[12] D.B. Kaplan and H. Georgi, Phys. Lett. 136B, 183 (1984); H. Georgi, ibid. 151B, 57 (1985).

[13] J. F. Gunion, H. E. Haber, G. L. Kane, and S. Dawson, SCIPP89/13; J.F. Gunion, H.E. Haber, G.L. Kane, and S. Dawson, errata, hep-ph/9302272; F. Boudjema and A. Semenov, Phys. Rev. D 66, 095007 (2002).

[14] B.W. Lee, C. Quigg, and H.B. Thacker, Phys. Rev. Lett. 38, 883 (1977); B.W. Lee, C. Quigg, and H.B. Thacker, Phys. Rev. D 16, 1519 (1977).

[15] M. Battaglia, E. Boos, and W.M. Yao, in Proceedings of the APS/DPF/DPB Summer Study on the Future of Particle Physics (Snowmass 2001), edited by R. Davidson and C. Quigg, hep-ph/0111276.

[16] D.A. Dicus, C. Kao, and S.S. Willenbrock, Phys. Lett. B 203, 457 (1988); E.W. Glover and J.J. van der Bij, Nucl. Phys. B309, 282 (1988); T. Plehn, M. Spira, and P.M. Zerwas, ibid. B479, 46 (1996); B531, 655(E) (1998).

[17] A. Djouadi, W. Kilian, M. Mühlleitner, and P.M. Zerwas, Eur. Phys. J. C 10, 27 (1999); D.J. Miller and S. Moretti, ibid. 13, 459 (2000).

[18] C. Castanier, P. Gay, P. Lutz, and J. Orloff, hep-ex/0101028.

[19] F. Boudjema and E. Chopin, Z. Phys. C 73, 85 (1996); V.A. Ilyin, A.E. Pukhov, Y. Kurihara, Y. Shimizu, and T. Kaneko, Phys. Rev. D 54, 6717 (1996).

[20] F. Gianotti et al., hep-ph/0204087.

[21] U. Baur, T. Plehn, and D. Rainwater, Phys. Rev. Lett. 89, 151801 (2002).

[22] A. Blondel, A. Clark, and F. Mazzucato, ATL-PHYS-2002029, 2002.

[23] T. Sjostrand, P. Eden, C. Friberg, L. Lönnblad, G. Miu, S. Mrenna, and E. Norrbin, Comput. Phys. Commun. 135, 238 (2001).

[24] G. Ambrosio et al., Fermilab-TM-2149, 2001.

[25] A. Djouadi, W. Kilian, M. Mühlleitner, and P.M. Zerwas, Eur. Phys. J. C 10, 45 (1999).

[26] A. Dobrovolskaya and V. Novikov, Z. Phys. C 52, 427 (1991); D.A. Dicus, K.J. Kallianpur, and S.S. Willenbrock, Phys. Lett. B 200, 187 (1988); A. Abbasabadi, W.W. Repko, D.A. Dicus, and R. Vega, ibid. 213, 386 (1988); W.Y. Keung, Mod. Phys. Lett. A 2, 765 (1987).
[27] V.D. Barger, T. Han, and R.J. Phillips, Phys. Rev. D 38, 2766 (1988).

[28] U. Baur, T. Plehn, and D. Rainwater (in preparation); see also R. Lafaye, D.J. Miller, M. Mühlleitner, and S. Moretti, hep-ph/0002238.

[29] E.W. Glover, J. Ohnemus, and S.S. Willenbrock, Phys. Rev. D 37, 3193 (1988).

[30] S. Dawson, S. Dittmaier, and M. Spira, Phys. Rev. D 58, 115012 (1998).

[31] M. Spira, A. Djouadi, D. Graudenz, and P.M. Zerwas, Nucl. Phys. B453, 17 (1995).

[32] K. Long, hep-ex/0212008, to appear in the Proceedings of the 31st International Conference on High Energy Physics, Amsterdam, The Netherlands, 2002.

[33] H.L. Lai et al., Phys. Rev. D 55, 1280 (1997).

[34] U. Baur et al., in Proceedings of the APS/DPF/DPB Summer Study on the Future of Particle Physics (Snowmass 2001), edited by R. Davidson and C. Quigg, hep-ph/0201227.

[35] T. Stelzer and W.F. Long, Comput. Phys. Commun. 81, 357 (1994); F. Maltoni and T. Stelzer, hep-ph/0208156.

[36] V.D. Barger, K.M. Cheung, T. Han, and R.J. Phillips, Phys. Rev. D 42, 3052 (1990).

[37] V.D. Barger, T. Han, J. Ohnemus, and D. Zeppenfeld, Phys. Rev. D 41, 2782 (1990).

[38] See, e.g., N. Kidonakis and G. Sterman, Nucl. Phys. B505, 321 (1997); R. Bonciani, S. Catani, M.L. Mangano, and P. Nason, ibid. B529, 424 (1998), and references therein.

[39] V. Barger and R.J.N. Phillips, Phys. Rev. Lett. 55, 2752 (1985); H. Baer, V. Barger, H. Goldberg, and R.J.N. Phillips, Phys. Rev. D 37, 3152 (1988); D.L. Rainwater, hep-ph/9908378.

[40] CDF Collaboration, F. Abe et al., Phys. Rev. Lett. 79, 584 (1997); Phys. Rev. D 56, 3811 (1997).

[41] G. Calucci and D. Treleani, Phys. Rev. D 57, 503 (1998); 60, 054023 (1999).

[42] M.L. Mangano, M. Moretti, F. Piccinini, R. Pittau, and A.D. Polosa, hep-ph/0206293.

[43] M. El Kacimi and R. Lafaye, ATL-PHYS-2002-015.

[44] M. Beneke et al., in Proceedings of the Workshop on "Standard model physics (and more) at the LHC," CERN-2000-04, edited by G. Altarelli and M. Mangano, hep-ph/0003033.

[45] Precision Higgs Working Group of Snowmass 2001 Collaboration, J. Conway, K. Desch, J.F. Gunion, S. Mrenna, and D. Zeppenfeld, in Proceedings of the APS/DPF/DPB Summer Study on the Future of Particle Physics (Snowmass 2001), edited by N. Graf, hep-ph/0203206.

[46] M. Mangano, "Monte Carlo tools for the LHC," theory seminar talk presented at CERN, Geneva, 2002, http:// mlm.home.cern.ch $/ \mathrm{mlm} /$

[47] U. Baur and E.L. Berger, Phys. Rev. D 47, 4889 (1993).

[48] R. W. Assman et al., CERN Report 2000-008; M. Battaglia, hep-ph/0103338. 